\title{
Mechanical Behaviors of Electrostatic Microbeams with Nonideal Supports
}

\author{
Yunxia Chang, ${ }^{1,2}$ Qichang Zhang $\mathbb{D},{ }^{1}$ Wei Wang, ${ }^{1}$ and Jianxin Han $^{3}$ \\ ${ }^{1}$ Tianjin Key Laboratory of Nonlinear Dynamics and Control, School of Mechanical Engineering, Tianjin University, \\ Tianjin 300350, China \\ ${ }^{2}$ National \& Local Joint Engineering Research Center of Metrology Instrument and System, \\ Department of Mechanical Engineering, Hebei University, Baoding 071000, China \\ ${ }^{3}$ Tianjin Key Laboratory of High Speed Cutting and Precision Machining, School of Mechanical Engineering, \\ Tianjin University of Technology and Education, Tianjin 300222, China
}

Correspondence should be addressed to Qichang Zhang; qzhang@tju.edu.cn

Received 30 November 2019; Revised 4 April 2020; Accepted 8 May 2020; Published 17 August 2020

Academic Editor: Ömer Cívalek

Copyright $(92020$ Yunxia Chang et al. This is an open access article distributed under the Creative Commons Attribution License, which permits unrestricted use, distribution, and reproduction in any medium, provided the original work is properly cited.

Deviation of the actual system from the ideal supporting conditions caused by micromachining errors and manufacturing defects or the requirement of innovative design and optimization of microelectromechanical systems (MEMS) make the nonideal boundary in the micro-/nanoresonator system receive wide attention. In this paper, we consider the neutral plane tension, fringing field, and nonideal boundary factors to establish a continuum model of electrostatically driven microbeam resonators. The convergent static solution with nine-order Galerkin decomposition is calculated. Then, based on the static solution, a 1-DOF dynamic equation of up to the fifth-order of the dynamic displacement using a Taylor expansion is derived. The method of multiple scales is used to study the effect of spring stiffness coefficients on the primary frequency response characteristics and hardening-softening conversion phenomena in four cases. The various law of the system's static and dynamic performances with the spring stiffness coefficients is obtained. The conditions for judging the hardening-softening transition are derived. So, adjusting the support stiffness values can be a measure of optimizing the resonator performance.

\section{Introduction}

Electrostatic microbeam resonators have the advantages of small size and light weight and are widely used in many fields, for instance, MEMS resonant sensor [1] and actuator [2]. Their small size allows sensitive systems to consume minimal energy and have low fabrication costs. However, due to the nonlinearity of the electrostatic force and the neutral plane tension, MEMS resonators can exhibit typical nonlinear dynamic characteristics. So far, the effects of nonlinearities on the static and dynamic performances of MEMS devices have been discussed in many works of literature [3-19].

But the above-mentioned works of literature only considered the ideal boundary conditions. In fact, both macro- and microstructures have errors and manufacturing defects, such as structure with elastically restrained, structure with nonuniform $[20,21]$, overcutting near anchor points [22], and initial deformation [23-26] of microstructures caused by residual stress. These cause the boundary conditions of the actual system to deviate from the ideal support conditions such that the displacement and rotation angle of the two fixed ends are not equal to zero. Bambill et al. [27] drew the conclusion that the characterization of real boundary conditions was much more important for microscale beams than for macroscale beams. In addition, Muthukumaran et al. [28] proposed that the boundary condition became one of the techniques responsible for the structural tuning of particular note in order to obtain a desired harmonic relation among its natural frequencies. Hence, the nonideal boundary has become a way of innovative design and optimization of electrostatic 
resonators [29]. Therefore, the nonideal boundary in the micro-/nanoresonator system has received wide attention from relevant scholars.

Rinaldi et al. [30] studied the boundary features of microcantilever beams through experiments. Results showed that nonclassical support boundaries could reduce the pull-in voltage of the microstructure. Rezaei et al. [31] analyzed the principal resonance behavior of piezoelectric actuated microcantilever and clamped beam under nonideal boundary conditions by using a two-dimensional multiscale method, and obtained the influence of nonideal boundary on the amplitude and frequency. Zhong et al. [32, 33] calculated the equivalent stiffness of nonideal support with fixed microbeam and microcantilever beam, respectively, and obtained the influence of nonideal boundary on vibration mode and frequency. Alkharabsheh and Younis [24] studied the fixed microarches with nonideal boundaries and compared the numerical results with experiments. Results demonstrated that nonideal boundary conditions had a significant effect on the qualitative dynamical behavior of the MEMS arch such as lowering its natural frequencies and causing unpredictable snap through or dynamic pull-in. Ekici et al. [34] used a multiscale method to study the superharmonic and subharmonic vibration of nonideal boundary microbeams. The nonideal boundary was simulated by helical spring. The conclusion was drawn that nonideal boundary conditions could cause shifting to the left or right side or no shifting in the graphs, depending on the mode numbers, axial force, deflections, and moments on the boundaries. Zeng et al. [35] studied the simulation of the nonideal boundary of the inclined supported beam. The finite element method and Galerkin method were used to study the static characteristics of the microbeam, which was in good agreement with the experimental results.

In recent years, the static and dynamic characteristics of microbeam were studied by considering nonideal support along with edge field, or other factors. In Pallay and Towfighian's literature [29], a MEMS-parametric resonator was introduced, which used nonideal supports and electrostatic edge fields to achieve an innovative design of energy-efficient resonators. Bashma et al. [36] used the finite element method based on wavelet transform to obtain the influence of nonideal support and edge effect on static attraction voltage and first-order natural frequency of the microcantilever beam. Chuang et al. [37] obtained an approximate analytical solution to the pull-in voltage of a microcurled cantilever beam considering the nonideal boundary and edge effect. Yayli [38] investigated the lateral free vibration of microbeams under various boundary conditions on the basis of the gradient theory of elasticity. Lishchynska et al. [39] established a model for predicting the static behavior of an electrostatic microcantilever beam considering the residual stress gradient and nonideal anchors. The analytical results were in good agreement with the experimental results. Tadi Beni et al. [40] introduced the modified couple stress theory, in conjunction with the MAD solving method, to investigate the effect of the Casimir attraction, Elastic boundary conditions, and size dependency on nonlinear pull-in behavior of the supported beam. Shojaeian et al. [41] studied the electromechanical instabilities of micro-/nanobeams with an initial curved shape and subjected to the electrostatic field and Casimir intermolecular force using a modified couple stress theory. However, they seldom analyze their effects on dynamic response.

The purpose of this article is to explore the influence law of nonideal boundaries on the dynamics of resonators and to provide theoretical support for the optimal design of MEMS. In this paper, a continuum model of electrostatic microbeam resonators is established. The convergent static solution with nine-order Galerkin decomposition [42] is calculated. It is used to investigate the influences of nonideal boundaries on the system's static characteristics. Based on the static solution, a 1-DOF dynamic equation of up to five orders of the dynamic displacement using Taylor expansion is derived. This quintic equation is a powerful complement to the cubic equation. Then, the method of multiple scales [43] is used to study the effects of nonideal boundaries on the primary frequency response characteristics and hardening-softening conversion phenomena in four cases. The various law of the system's static and dynamic performances with the spring stiffness coefficients is obtained. The details are going to be discussed in the following sections.

The paper is organized as follows: an introduction including the literature review and motivation of the research, equation of motion and methods, results and discussion, and lastly, conclusions.

\section{Equation of Motion and Methods}

Due to micromachining errors and manufacturing defects or the requirement of innovative design, the displacements and rotation angles of the two fixed ends of the microbeam are not equal to zero. To simulate and quantify these small rotation angles and deflections, artificial rotational and translational springs are introduced [24, 30-33, 44]. In this paper, only symmetrical springs are considered. That is, the stiffness coefficients of left and right springs, $K_{R}$ and $K_{T}$, are the same. The schematic diagram is shown in Figure 1. With the application of DC and AC voltage across the beam and electrode backplate, a distributed electrostatic force between the beam and electrode is generated to deform and vibrate the microbeam.

The first-order fringing field correction of electrostatic force per unit length, namely, Palmer model [45, 46], is expressed as follows:

$$
F_{\mathrm{ef}}=0.65 \cdot \frac{g_{0}-\widehat{w}}{b_{0}} \cdot \frac{\varepsilon_{0} b_{0}\left[V_{\mathrm{D}}+V_{\mathrm{A}} \sin (\widehat{\Omega} \cdot \widehat{t})\right]^{2}}{2\left(g_{0}-\widehat{w}\right)^{2}} .
$$

Hence, the governing equation per unit length along the microbeam [24, 32, 42, 47] can be given by the following equation: 


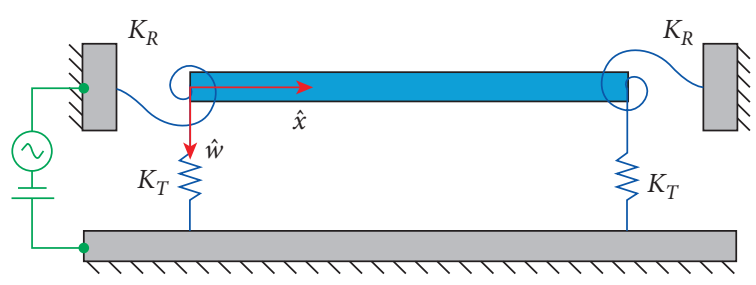

FIGURE 1: Schematic diagram of one-electrode microbeam with nonideal boundary condition.

$$
\widehat{E} I \frac{\partial^{4} \widehat{w}}{\partial \widehat{x}^{4}}+\widehat{\mu} \frac{\partial \widehat{w}}{\partial \widehat{t}}+\rho b_{0} h_{0} \frac{\partial^{2} \widehat{w}}{\partial \hat{t}^{2}}=\left[\widehat{N}+\frac{\widehat{E} b_{0} h_{0}}{2 l_{0}} \int_{0}^{l_{0}}\left(\frac{\partial \widehat{w}}{\partial \widehat{x}}\right)^{2} \mathrm{~d} \widehat{x}\right] \frac{\partial^{2} \widehat{w}}{\partial \widehat{x}^{2}}+\frac{\varepsilon_{0} b_{0}\left[V_{\mathrm{D}}+V_{\mathrm{A}} \sin (\widehat{\Omega} \cdot \widehat{t})\right]^{2}}{2\left(g_{0}-\widehat{w}\right)^{2}}\left(1+0.65 \cdot \frac{g_{0}-\widehat{w}}{b_{0}}\right)
$$

where $\widehat{x}$ is the position along the microbeam length, $w(\widehat{x}, \hat{t})$ represents transverse deflection, $I$ is the moment of inertia of the cross section $\left(I=b_{0} h_{0}^{3} / 12\right.$, where $b_{0}$ and $h_{0}$ represent, respectively, the microbeam's width and thickness), $l_{0}$ and $g_{0}$ are, respectively, the microbeam's length and the initial gap, $\widehat{\mu}$ and $\rho$ represent, respectively, the viscous damping per unit length and the material density, $\varepsilon_{0}$ is the dielectric constant of the gap medium, $\widehat{t}$ is time, $\widehat{t}$ represents the effective Young's modulus with $\widehat{E}=E /\left(1-v^{2}\right)$ for a wide microbeam $\left(b_{0} \geq 5 h_{0}\right)$ and $\widehat{E}=E$ for a narrow microbeam $\left(b_{0}<5 h_{0}\right)$, in which $\widehat{E}$ represents Young's modulus while $v$ represents the Poisson's ratio.

The system's nonideal boundary conditions are given by the following equation:

$$
\begin{aligned}
& \text { at } \widehat{x}=0 \text { : } \\
& \widehat{E} I \frac{\partial^{2} \widehat{w}}{\partial \widehat{x}^{2}}-K_{\mathrm{R}} \frac{\partial \widehat{w}}{\partial \widehat{x}}=0, \\
& \widehat{E} I \frac{\partial^{3} \widehat{w}}{\partial \widehat{x}^{3}}-\widehat{N} \frac{\partial \widehat{w}}{\partial \widehat{x}}+K_{\mathrm{T}} \widehat{w}=0, \\
& \text { at } \widehat{x}=l_{0} \text { : } \\
& \widehat{E} I \frac{\partial^{2} \widehat{w}}{\partial \widehat{x}^{2}}+K_{\mathrm{R}} \frac{\partial \widehat{w}}{\partial \widehat{x}}=0, \\
& \widehat{E} I \frac{\partial^{3} \widehat{w}}{\partial \widehat{x}^{3}}-\widehat{N} \frac{\partial \widehat{w}}{\partial \widehat{x}}-K_{\mathrm{T}} \widehat{w}=0,
\end{aligned}
$$

where $K_{\mathrm{R}}$ and $K_{\mathrm{T}}$ represent the rotational and translational stiffness.

For convenience, introduce the following nondimensional variables:

$$
w=\frac{\widehat{w}}{g_{0}}, x=\frac{\widehat{x}}{l_{0}}, t=\frac{\widehat{t}}{\mathrm{~T}}
$$

where $\mathrm{T}=\sqrt{\rho b_{0} h_{0} l_{0}^{4} /(\widehat{E} I)}$. Thus, the governing equation of motion and the system's boundary conditions become as follows:

$$
\begin{aligned}
\frac{\partial^{4} w}{\partial x^{4}}+\mu \frac{\partial w}{\partial t}+\frac{\partial^{2} w}{\partial t^{2}}= & {\left[N+\alpha_{1} \int_{0}^{1}\left(\frac{\partial w}{\partial x}\right)^{2} \mathrm{~d} x\right] \frac{\partial^{2} w}{\partial x^{2}} } \\
& +\frac{\alpha_{2}\left[V_{D}+V_{A} \sin (\Omega \cdot t)\right]^{2}}{(1-w)^{2}}[1+\eta(1-w)],
\end{aligned}
$$

at $x=0$ :

$$
\begin{aligned}
& \frac{\partial^{2} w}{\partial x^{2}}-\kappa_{\mathrm{R}} \frac{\partial w}{\partial x}=0 \\
& \frac{\partial^{3} w}{\partial x^{3}}-N \frac{\partial w}{\partial x}+\kappa_{\mathrm{T}} w=0,
\end{aligned}
$$

at $x=1$ :

$$
\begin{aligned}
& \frac{\partial^{2} w}{\partial x^{2}}+\kappa_{\mathrm{R}} \frac{\partial w}{\partial x}=0, \\
& \frac{\partial^{3} w}{\partial x^{3}}-N \frac{\partial w}{\partial x}-\kappa_{\mathrm{T}} w=0,
\end{aligned}
$$

where $\quad \widehat{\mu}=\widehat{\mu} l_{0}^{4} /(\widehat{E} I T), \quad N=\widehat{N} l_{0}^{2} /(\widehat{E} I), \quad \Omega=\widehat{\Omega} / T, \quad \alpha_{1}=6$ $\left(g_{0} / h_{0}\right)^{2}, \alpha_{2}=6 \varepsilon_{0} l_{0}^{4} /\left(\widehat{E} h_{0}^{3} t g_{0}^{3}\right), \eta=0.65 \cdot g_{0} / b_{0}, \kappa_{\mathrm{R}}=K_{\mathrm{R}} l_{0} /$ $(\widehat{E} I), \kappa_{\mathrm{T}}=K_{\mathrm{T}} l_{0}^{3} /(\widehat{E} I)$.

Galerkin method is a powerful method, which is capable of handling static and dynamic problems of nonlinear systems. It is limited by solving the mode shapes. See Appendix A for the expression of the mode shapes.

In a microbeam resonator, the DC voltage causes the microbeam to form a new static equilibrium position, and the $\mathrm{AC}$ voltage excites the microbeam to vibrate around the equilibrium position. Thus, microbeam deflection can be given by the following equation:

$$
w(x, t)=w_{\mathrm{s}}(x)+w_{\mathrm{d}}(x, t)
$$

where the static deflection due to the DC voltage is denoted by $w_{\mathrm{s}}(x)$, and the dynamic deflection due to the AC voltage is denoted by $w_{\mathrm{d}}(x, t)$.

To calculate the static deflection, set the time-varying terms in (5) equal to zero and obtain the following: 
$\frac{\partial^{4} w_{\mathrm{s}}}{\partial x^{4}}=\left[N+\alpha_{1} \int_{0}^{1}\left(\frac{\partial w_{\mathrm{s}}}{\partial x}\right)^{2} \mathrm{~d} x\right] \frac{\partial^{2} w_{\mathrm{s}}}{\partial x^{2}}+\frac{\alpha_{2}\left[V_{\mathrm{D}}\right]^{2}}{\left(1-w_{\mathrm{s}}\right)^{2}}\left[1+\eta\left(1-w_{\mathrm{s}}\right)\right]$

Here the static deflection is expressed as $w_{\mathrm{s}}=\sum A_{\mathrm{s}, i} \phi_{i}(x), i=1,2,3, L \ldots$, where $A_{\mathrm{s}, i}$ is the $i$ th generalized coordinate (here is a constant), $\phi_{i}(x)$ is the $i$ th mode shape, and $i$ is the order of Galerkin discreteness. Substituting the expression into (9) and applying the Galerkin method yield the system of nonlinear algebraic equations.

Substituting the above (8) and (9) into (5), setting $V_{\mathrm{D}} \gg V_{\mathrm{A}}$, omitting the parameter exciting terms, and keeping the fifth power terms about $w_{\mathrm{d}}$, yield the following:

$$
\begin{aligned}
\frac{\partial^{4} w_{\mathrm{d}}}{\partial x^{4}}+\frac{\partial^{2} w_{\mathrm{d}}}{\partial t^{2}}+\mu \frac{\partial w_{\mathrm{d}}}{\partial t}= & \left(\alpha_{1} \int_{0}^{1}\left(\frac{\partial w_{\mathrm{s}}}{\partial x}\right)^{2} \mathrm{~d} x+N\right) \frac{\partial^{2} w_{\mathrm{d}}}{\partial x^{2}}+2 \alpha_{1} \frac{\partial^{2} w_{\mathrm{s}}}{\partial x^{2}} \int_{0}^{1} \frac{\partial w_{\mathrm{s}}}{\partial x} \frac{\partial w_{\mathrm{d}}}{\partial x} \mathrm{~d} x \\
& +\frac{\alpha_{2}\left[V_{\mathrm{D}}\right]^{2}\left[2+\eta\left(1-w_{\mathrm{s}}\right)\right]}{\left(1-w_{\mathrm{s}}\right)^{3}} w_{\mathrm{d}}+\alpha_{1} \frac{\partial^{2} w_{\mathrm{s}}}{\partial x^{2}} \int_{0}^{1}\left(\frac{\partial w_{\mathrm{d}}}{\partial x}\right)^{2} \mathrm{~d} x+2 \alpha_{1} \frac{\partial^{2} w_{\mathrm{d}}}{\partial x^{2}} \int_{0}^{1} \frac{\partial w_{\mathrm{s}}}{\partial x} \frac{\partial w_{\mathrm{d}}}{\partial x} \mathrm{~d} x \\
& +\frac{\alpha_{2}\left[V_{\mathrm{D}}\right]^{2}\left[3+\eta\left(1-w_{\mathrm{s}}\right)\right]}{\left(1-w_{\mathrm{s}}\right)^{4}} w_{\mathrm{d}}^{2}+\alpha_{1} \frac{\partial^{2} w_{\mathrm{d}}}{\partial x^{2}} \int_{0}^{1}\left(\frac{\partial w_{\mathrm{d}}}{\partial x}\right)^{2} \mathrm{~d} x+\frac{\alpha_{2}\left[V_{\mathrm{D}}\right]^{2}\left[4+\eta\left(1-w_{\mathrm{s}}\right)\right]}{\left(1-w_{\mathrm{s}}\right)^{5}} w_{\mathrm{d}}^{3} \\
& +\frac{\alpha_{2}\left[V_{\mathrm{D}}\right]^{2}\left[5+\eta\left(1-w_{\mathrm{s}}\right)\right]}{\left(1-w_{\mathrm{s}}\right)^{6}} w_{\mathrm{d}}^{4}+\frac{\alpha_{2}\left[V_{\mathrm{D}}\right]^{2}\left[6+\eta\left(1-w_{\mathrm{s}}\right)\right]}{\left(1-w_{\mathrm{s}}\right)^{7}} w_{\mathrm{d}}^{5} \\
& +\frac{2 \alpha_{2} V_{\mathrm{D}} V_{\mathrm{A}}\left[1+\eta\left(1-w_{\mathrm{s}}\right)\right]}{\left(1-w_{\mathrm{s}}\right)^{2}} \sin (\Omega t) .
\end{aligned}
$$

Omitting the dynamic forcing term, neglecting damping term, and keeping only linear terms about $w_{\mathrm{d}}$ in (10) yield the following:

$$
\frac{\partial^{4} w_{\mathrm{d}}}{\partial x^{4}}+\frac{\partial^{2} w_{\mathrm{d}}}{\partial t^{2}}=N \frac{\partial^{2} w_{\mathrm{d}}}{\partial x^{2}}+\alpha_{1} \frac{\partial^{2} w_{\mathrm{s}}}{\partial x^{2}} \int_{0}^{1}\left(2 \frac{\partial w_{\mathrm{s}}}{\partial x} \frac{\partial w_{\mathrm{d}}}{\partial x}\right) \mathrm{d} x+\alpha_{1} \frac{\partial^{2} w_{\mathrm{d}}}{\partial x^{2}} \int_{0}^{1}\left(\frac{\partial w_{\mathrm{s}}}{\partial x}\right)^{2} \mathrm{~d} x+\frac{\alpha_{2}\left[V_{\mathrm{D}}\right]^{2}\left[2+\eta\left(1-w_{\mathrm{s}}\right)\right]}{\left(1-w_{\mathrm{s}}\right)^{3}} w_{\mathrm{d}}
$$

Let $w_{\mathrm{d}}(x, t)=\phi(x) e^{i \oplus t}$, where $\phi(x)$ is mode shape and $\omega$ is the frequency of the system. Then, one can obtain the frequency of the system for various conditions.

\section{Results and Discussion}

Next, this paper analyzes the static and dynamic characteristics of the systems.

3.1. Static Analysis. In this section, the influences of the support spring coefficients on the static deflection and the natural frequency of the system are discussed by the numerical solution.

3.1.1. Convergence Analysis. Because the microbeam maintains symmetric shapes during motion, only symmetric modes (namely the modes when $i$ equals even number) are considered to verify the convergence using the data in literature [4].

Setting $\kappa_{\mathrm{R}}=+\infty, \kappa_{\mathrm{T}}=+\infty$ regardless of fringing field effect, the outcome obtained by the first five symmetric modes (namely, $i=9$ ) is shown in Figure 2. Compared with Nayfeh et al. [4], the convergence effect is good, and the research in this paper is feasible.

3.1.2. Static Deflection. Next, calculate the static deflection of a microbeam with the parameters listed in Table 1 and the various spring stiffness coefficients $\kappa_{\mathrm{R}}$ and $\kappa_{\mathrm{T}}$.

The effects of rotational stiffness on the system's static features are discussed by changing the value of rotational stiffness, as shown in Figure 3. The red dot dash line is the corresponding stable value when the rotational stiffness $\kappa_{\mathrm{R}}$ is infinite. The deflection-DC voltage curve is divided into the upper branch and lower one. The solid line of the lower branch represents the steady state, and the black 
TABle 1: Physical parameters of microbeam-based resonator [4].

\begin{tabular}{lc}
\hline Parameters & Values \\
\hline Young's modulus $E(\mathrm{GPa})$ & 166 \\
Density $\rho\left(\mathrm{kg} / \mathrm{m}^{3}\right)$ & 2332 \\
Poisson's ratio $v$ & 0.06 \\
Length $l_{0}(\mu \mathrm{m})$ & 510 \\
Width $b_{0}(\mu \mathrm{m})$ & 100 \\
Thickness $h_{0}(\mu \mathrm{m})$ & 1.5 \\
The dielectric constant $\varepsilon_{0}(\mathrm{~F} / \mathrm{m})$ & $8.854 \times 10^{-12}$ \\
Initial gap width $g_{0}(\mu \mathrm{m})$ & 1.18 \\
\hline
\end{tabular}

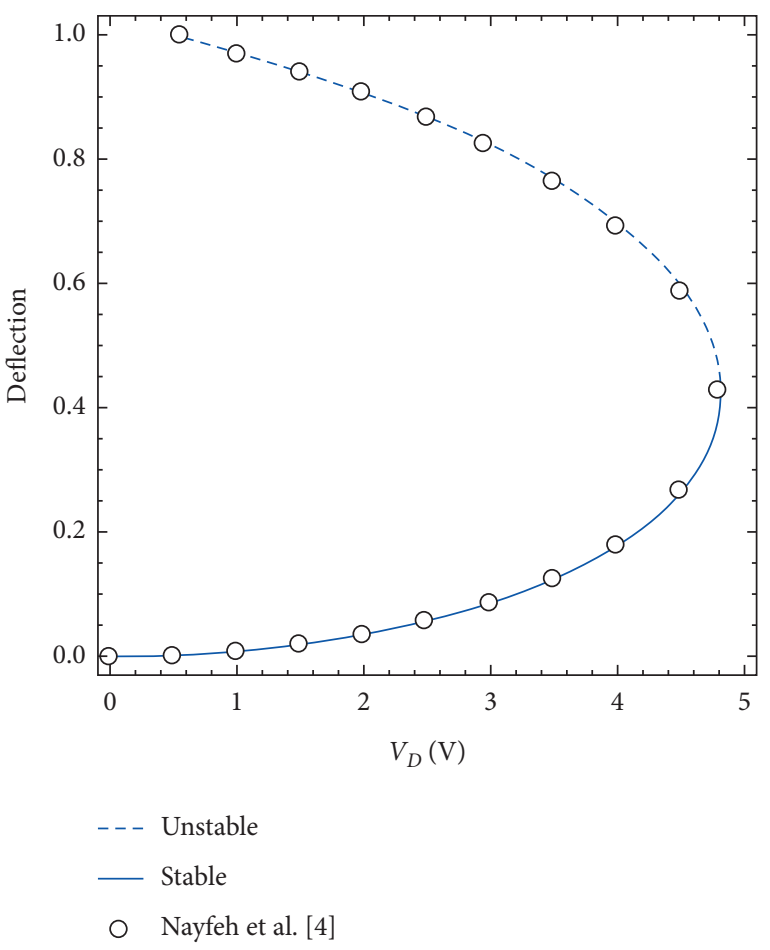

Figure 2: Maximum static deflection of a microbeam-based resonator.

dotted line of the upper branch represents the unstable state. Only the stable values make sense. With the increase of $\kappa_{\mathrm{R}}$ before pull-in phenomenon occurs, the static deflection corresponding to the same voltage decreases, the static pull-in voltage increases and eventually reaches the stable value.

Figure 4 shows the effect of the translational stiffness on the static characteristics of the system. The red dot dash line is the corresponding stable value when the translational stiffness $\kappa_{\mathrm{T}}$ is infinite. As the $\kappa_{\mathrm{T}}$ goes up before pull-in phenomenon occurs, the static deflection corresponding to the same voltage goes down, the static pull-in voltage goes up, and eventually reaches the stable value.

In a word, with the growth of support stiffness (refers to rotational stiffness and translational stiffness) before pull-in phenomenon occurs, the static deflection corresponding to the same voltage goes down, the static pull-in voltage goes up, and eventually reaches a stable value.

3.1.3. Natural Frequency. The influence of the rotational coefficient $\kappa_{R}$ on the first-order frequency is shown in Figure 5. The red dot dash line is the corresponding stable value when the rotational stiffness $\kappa_{\mathrm{R}}$ is infinite. In the case of the same DC voltage, the greater the rotational stiffness is, the higher the first-order frequency is until it finally tends to the stable value.

Figure 6 shows the effect of translational stiffness $\kappa_{\mathrm{T}}$ on first-order frequency by applying the numerical method. The red dot dash line is the corresponding value when the 


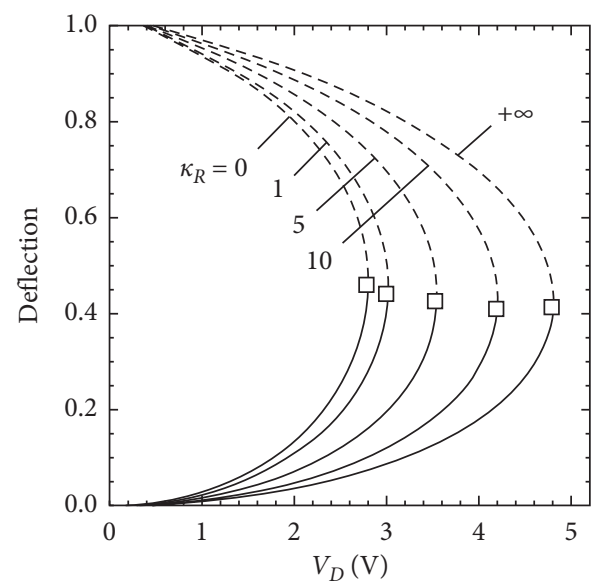

$\square$ Pull-in position

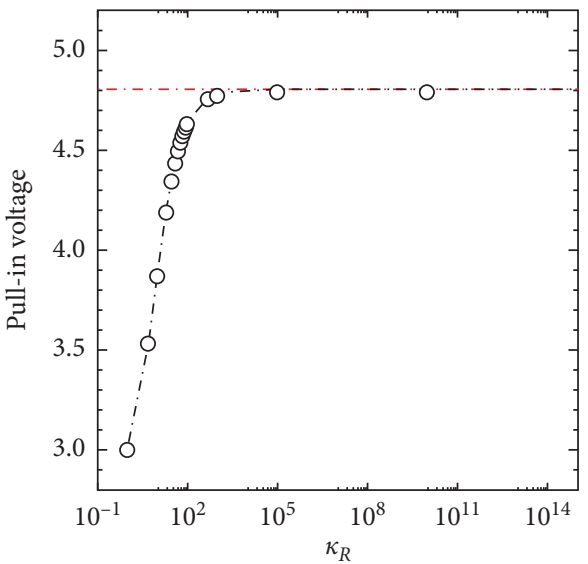

(b)

FIGURE 3: The effect of rotational stiffness on static features by applying the first five-order symmetry modes. (a) Relationship between maximum static deflection and DC voltage under different rotational stiffness. (b) Variation trend of the pull-in voltage with rotational stiffness.

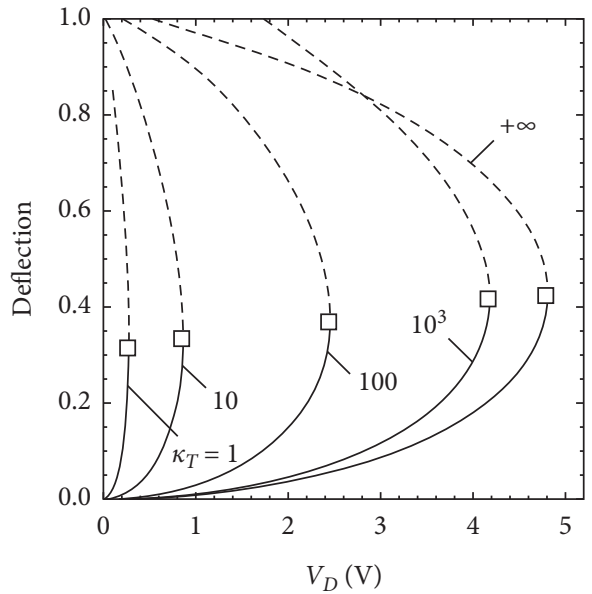

(a)

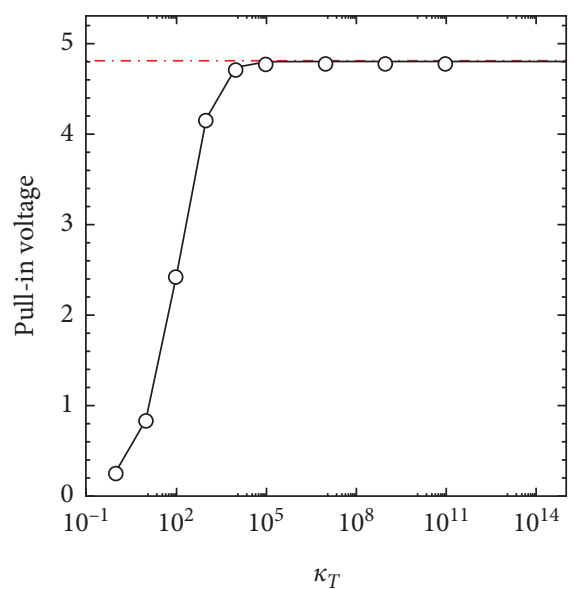

(b)

Figure 4: The effect of translational stiffness on static features by applying the first five-order symmetry modes. (a) Relationship between maximum displacement and DC voltage under different translational stiffness. (b) Variation trend of the pull-in voltage with translational stiffness.

translational stiffness $\kappa_{\mathrm{T}}$ is infinite. In the case of the same DC voltage, the greater the translational stiffness is, the higher the first-order frequency is until it eventually tends to the stable value.

In short, as the support stiffness (refers to rotational stiffness and translational stiffness) goes up, the first-order natural frequency increases, until it eventually tends to the stable value.
3.2. Dynamic Analysis. Introducing $w_{\mathrm{d}}(x, t) \approx u(t) \cdot \phi_{1}(x)$ and applying the Galerkin method, obtain the following dynamic equation via equation (10):

$$
\ddot{u}+\mu \dot{u}+\omega_{n}^{2} u+k_{2} u^{2}+k_{3} u^{3}+k_{4} u^{4}+k_{5} u^{5}=f_{1} \sin (\Omega t),
$$

where $\omega_{n}^{2}=k_{1}$, 


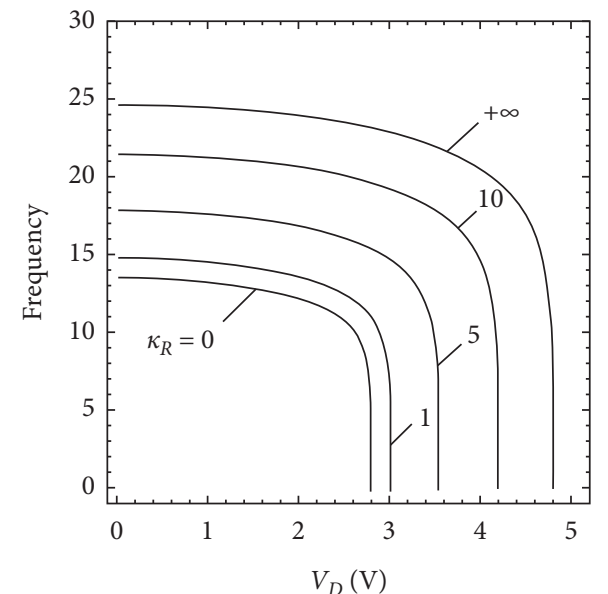

(a)

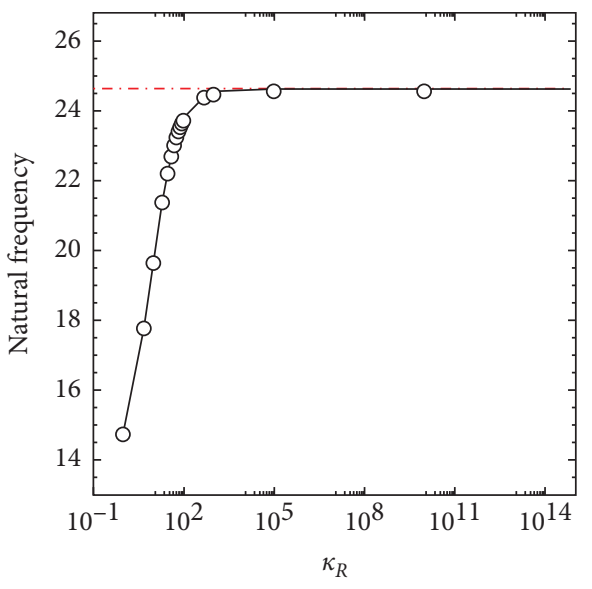

(b)

FiguRE 5: The effect of rotational stiffness on first-order frequency natural frequency. (a) Relationship between natural frequency and DC voltage under different rotational stiffness. (b) Variation trend of the natural frequency with rotational stiffness.

$$
\begin{aligned}
& k_{1}=\frac{\int_{0}^{1} \phi_{1}\left[\phi_{1}^{(4)}-N \cdot \phi_{1}^{(2)}-2 \alpha_{1} w_{\mathrm{s}}^{(2)} \int_{0}^{1} w_{\mathrm{s}}^{(1)} \phi_{1}^{(1)} \mathrm{d} x-\alpha_{1} \phi_{1}^{(2)} \int_{0}^{1}\left(w_{\mathrm{s}}^{(1)}\right)^{2} \mathrm{~d} x-\left(\left(\alpha_{2} V_{\mathrm{D}}^{2}\left(2+\eta-\eta \cdot w_{\mathrm{s}}\right) \phi_{1}\right) /\left(1-w_{\mathrm{s}}\right)^{3}\right)\right] \mathrm{d} x}{\int_{0}^{1} \phi_{1}^{2} \mathrm{~d} x}, \\
& k_{2}=\frac{\int_{0}^{1} \phi_{1}\left[-\left(\left(\alpha_{2} V_{\mathrm{D}}^{2}\left(3+\eta-\eta \cdot w_{\mathrm{s}}\right) \phi_{1}\right) /\left(1-w_{\mathrm{s}}\right)^{4}\right)-\alpha_{1} w_{\mathrm{s}}^{(2)} \int_{0}^{1}\left(\phi_{1}^{(1)}\right)^{2} \mathrm{~d} x-2 \alpha_{1} \phi_{1}^{(2)} \int_{0}^{1}\left(w_{\mathrm{s}}^{(1)} \phi_{1}^{(1)}\right) \mathrm{d} x\right] \mathrm{d} x}{\int_{0}^{1} \phi_{1}^{2} \mathrm{~d} x}, \\
& k_{3}=\frac{\int_{0}^{1} \phi_{1}\left[-\left(\left(\alpha_{2} V_{\mathrm{D}}^{2}\left(4+\eta-\eta \cdot w_{\mathrm{s}}\right) \phi_{1}\right) /\left(1-w_{\mathrm{s}}\right)^{5}\right)-\alpha_{1} \phi_{1}^{(2)} \int_{0}^{1}\left(\phi_{1}^{(1)}\right)^{2} \mathrm{~d} x\right] \mathrm{d} x}{\int_{0}^{1} \phi_{1}^{2} \mathrm{~d} x}, \\
& k_{4}=\frac{\int_{0}^{1} \phi_{1}\left[-\left(\left(\alpha_{2} V_{\mathrm{D}}^{2}\left(5+\eta-\eta \cdot w_{\mathrm{s}}\right) \phi_{1}\right) /\left(1-w_{\mathrm{s}}\right)^{6}\right)\right] \mathrm{d} x}{\int_{0}^{1} \phi_{1}^{2} \mathrm{~d} x}, \\
& k_{5}=\frac{\int_{0}^{1} \phi_{1}\left[-\left(\left(\alpha_{2} V_{\mathrm{D}}^{2}\left(6+\eta-\eta \cdot w_{\mathrm{s}}\right) \phi_{1}\right) /\left(1-w_{\mathrm{s}}\right)^{7}\right)\right] \mathrm{d} x}{\int_{0}^{1} \phi_{1}^{2} \mathrm{~d} x}, \\
& f_{1}=\frac{\int_{0}^{1}\left(\left(2 \alpha_{2} V_{\mathrm{D}} V_{\mathrm{A}}\left(1+\eta-\eta \cdot w_{\mathrm{s}}\right) \phi_{1}\right) /\left(1-w_{\mathrm{s}}\right)^{2}\right) \mathrm{d} x}{},
\end{aligned}
$$

$w_{\mathrm{s}}^{(j)}$ represents the corresponding Jth derivative of the function $w_{s}(x), \phi_{1}^{(j)}$ is the corresponding $J$ th derivative of the mode shape $\phi_{1}(x)$.

See Appendix B for the detailed solving process by the method of multiple scales.
Hence, frequency response equation is obtained as follows:

$$
a^{2}\left[\left(p_{1}-p_{3} a^{2}-p_{5} a^{4}\right)^{2}+\left(\frac{\mu}{2}\right)^{2}\right]=\left[\frac{f_{1}}{\left(2 \omega_{n}\right)}\right]^{2},
$$




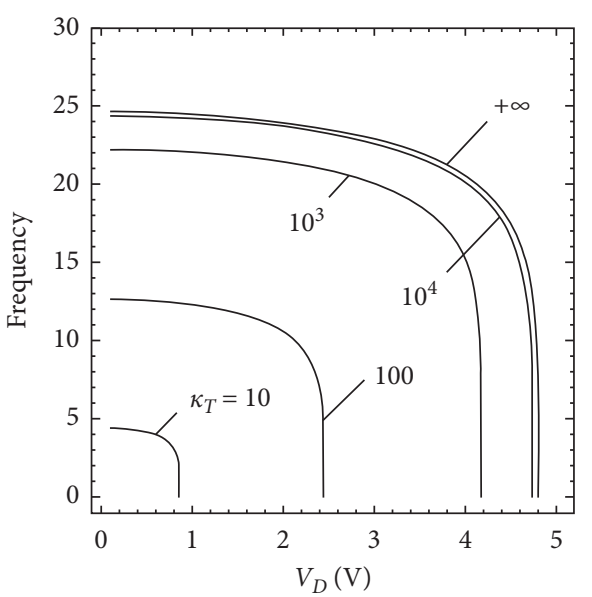

(a)

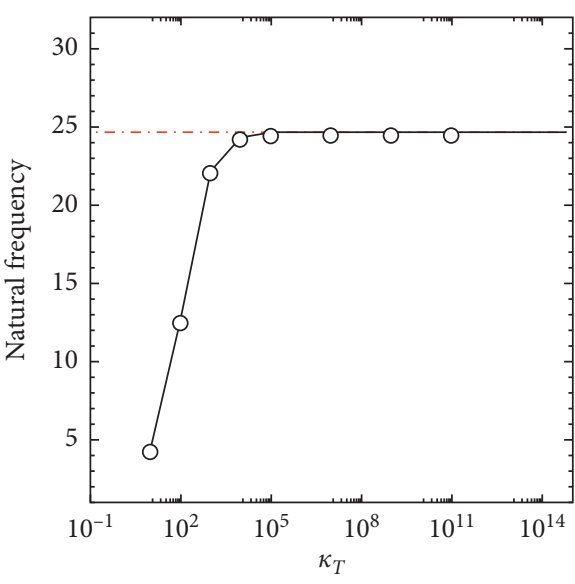

(b)

Figure 6: The effect of translational stiffness on first-order frequency fundamental frequency. (a) Relationship between natural frequency and DC voltage under different translational stiffness. (b) Variation trend of the natural frequency with translational stiffness.

where

$$
\begin{aligned}
& p_{1}=\left(\Omega-\omega_{n}\right), \\
& p_{3}=\frac{-\left(10 k_{2}^{2}-9 k_{3} \omega_{n}^{2}\right)}{24 \omega_{n}^{3}}, \\
& p_{5}=\frac{\left(-1940 k_{2}^{4}+6228 k_{2}^{2} k_{3} \omega_{n}^{2}-405 k_{3}^{2} \omega_{n}^{4}-6048 k_{2} k_{4} \omega_{n}^{4}+2160 k_{5} \omega_{n}^{6}\right)}{6912 \omega_{n}^{7}} .
\end{aligned}
$$

The peak amplitude is as follows:

$$
a_{\max }=\frac{f_{1}}{\mu \omega_{n}} .
$$

It is found through analysis that $a_{\max }$ is the function of DC voltage and AC voltage as independent variables for a given microbeam resonator.

The backbone curve is as follows:

$$
\omega_{c}=\omega_{n}+p_{3} a^{2}+p_{5} a^{4}
$$

When $p_{3}>0$, the nonlinearity has a hardening effect that tends to bend the frequency response curves to higher frequencies. When $p_{3}<0$, the nonlinearity has a softening effect, which tends to bend the frequency response curves to lower frequencies.

When $\left(\mathrm{d} \omega_{c} / \mathrm{d} a\right)=0$, a real threshold can be obtained as follows:

$$
a_{\mathrm{s}}=\sqrt{\frac{-p_{3}}{\left(2 p_{5}\right)}}
$$

If the real threshold $a_{\mathrm{s}}$ exists and satisfies the following condition, the system has a hardening-softening effect transition phenomenon.

$$
a_{\mathrm{s}} \leq a_{\max }
$$

3.2.1. Convergence Verification. Assuming that the maximum microbeam deflection is given by $w_{\max }=w_{\mathrm{s}}+\max \left(\phi_{1} \cdot u\right)$, where $w_{\mathrm{s}}$ is the approximate solution of the static deflection obtained by the nine-order Galerkin discretization. Given the voltage value, calculate the system's response, as shown in Figure 7.

It can be seen from Figure 7 that the regularity trend of the frequency response curve is consistent and the convergence is good. Therefore, the above-mentioned approximate solution of maximum microbeam deflection can be used to determine the vibration characteristics of the system.

3.2.2. Softening and Hardening Effects. Nonlinear hardening and softening characters have been reported in many works of literature. The system's nonlinear softening and hardening characteristics are determined by parameter $p_{3}$, which is determined by the DC voltage $V_{\mathrm{D}}$, static deflection $w_{\mathrm{s}}$, and natural frequency $\omega_{n}$. Furtherly, since the static displacement and natural frequency depend on $V_{\mathrm{D}}$ and the boundary support stiffness values, the parameter $p_{3}$ is ultimately affected by the DC voltage and the boundary support stiffness values. So, the boundary support stiffness values do affect the softening and hardening characteristics of the system. 


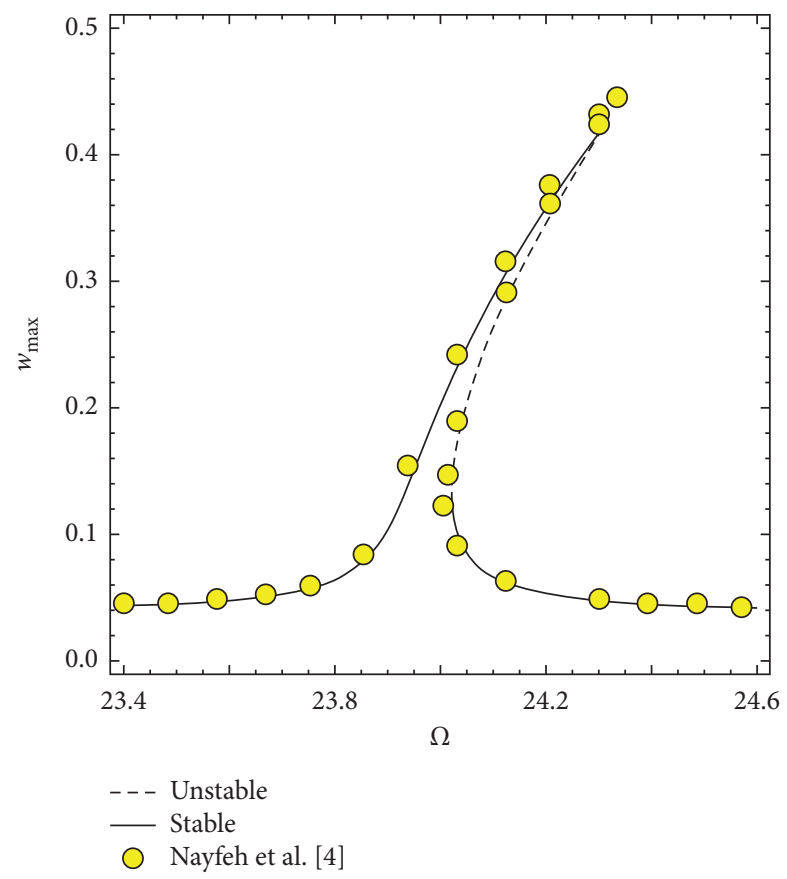

FiguRE 7: Convergence verification of frequency response curve regardless of the fringing field effect: $\kappa_{\mathrm{R}}=+\infty, \kappa_{\mathrm{T}}=+\infty, V_{\mathrm{D}}=2 \mathrm{~V}$, $V_{\mathrm{A}}=0.01 \mathrm{~V}$.

To investigate how the nonideal support stiffness to affect the system's frequency response curve in this section, there are four cases to discuss as seen in Table 2 .

(1) Comparison of Case 1 and Case 2. The stiffness values in Case 1 are used to simulate the ideal fixed boundary system, and the data in Case 2 simulate a nonideal boundary system. Other physical parameters refer to Table 1 .

For a given electrostatic microbeam resonator in Cases 1 and 2 , the parameter $p_{3}$ is the function of the DC voltage. Hence, Figure 8(a) shows the relationship between the parameter $p_{3}$ and DC voltage $V_{\mathrm{D}}$ under different boundary conditions. As the DC voltage increases, the parameter $p_{3}$ decreases. The dotted line $p_{3}=0$ is the boundary of the hardening and softening features. As shown in Figure 8(a), when $V_{\mathrm{D}}=2 \mathrm{~V}$, hardening characteristics that ideal boundary system demonstrates are predicted. According to Figure 7, this prediction is true. Meanwhile, the nonideal boundary system at the same DC voltage also shows hardening characteristics.

It can be seen clearly from Figure 8(b) that the nonideal boundary has an influence on the softening and hardening features. The abscissa value corresponding to the intersection point $S_{1}$ is smaller than the value of $S_{0}$. Approximate maximum deflection at the time of the transition can be obtained according to the formula $w_{\text {max }, \mathrm{s}} \approx \max \left(w_{\max }+\phi_{1} a_{\mathrm{s}}\right)$, as shown in Figure 9. Obviously, the nonideal boundary reduces the critical deflection of the transition. Therefore, under the same DC voltage, the transition occurs first.

For the sake of illustration, select the three groups $V_{\mathrm{D}}$ corresponding to the dotted line in the above Figure $8(\mathrm{~b})$ to analyze the amplitude-frequency response. Assume that the dimensionless damping coefficient is $\mu=0.01$.

When $V_{\mathrm{D}}<S_{1}$, the real threshold $a_{\mathrm{s}}$ of the ideal boundary system exists, and the system is in hardening effect region. Nevertheless, whether there is a hardening-softening effect, conversion needs to be furtherly analyzed and judged by the second condition of equation (19). When $V_{\mathrm{D}}$ is given, the system's excitation amplitude $f_{1}$ depends on the AC voltage $V_{\mathrm{A}}$, so the parameter $a_{\max }$ in equation (19) is actually determined by the parameter $V_{\mathrm{A}}$. Therefore, given a suitable $V_{\mathrm{A}}$ to meet with the condition of equation (19), there is a hardening-softening effect conversion in the ideal boundary system, as shown in Figure 10(b). In this case, the nonideal boundary system has a hardening-softening conversion phenomenon, but the maximum deflection of the transition is less than that of the ideal boundary system, as shown in Figure 10(a).

When $S_{1}<V_{\mathrm{D}}<S_{0}$, the two boundary systems behave differently. The real threshold $a_{\mathrm{s}}$ of the ideal boundary system exists, and there is a hardening-softening conversion if a suitable $V_{\mathrm{A}}$ to meet with the condition of equation (19) is selected, as shown in Figure 11(b). While the real threshold $a_{\mathrm{s}}$ of the nonideal boundary system does not exist, the system shows a softening effect and there is not a hardeningsoftening conversion, as shown in Figure 11(a).

When $V_{\mathrm{D}}>S_{0}$, the real threshold $a_{\mathrm{s}}$ of both boundary systems do not exist. So there are no hardening-softening conversion phenomena in both boundary systems. Both boundary systems show a softening effect, as shown in Figure 12.

In summary, the laws of the dynamic performance of the system with the spring stiffness coefficients are obtained. 
TABLe 2: Analysis cases.

\begin{tabular}{lcc}
\hline & Torsional spring stiffness value $\kappa_{\mathrm{R}}$ & Translational spring stiffness value $\kappa_{\mathrm{T}}$ \\
\hline Case 1 & $10^{50}(+\infty)$ & $10^{50}(+\infty)$ \\
Case 2 & $10^{2}$ & $10^{4}$ \\
Case 3 & $10^{50}(+\infty)$ & $10^{3}, 10^{4}$ \\
Case 4 & 1,5 & $10^{50}(+\infty)$ \\
\hline
\end{tabular}

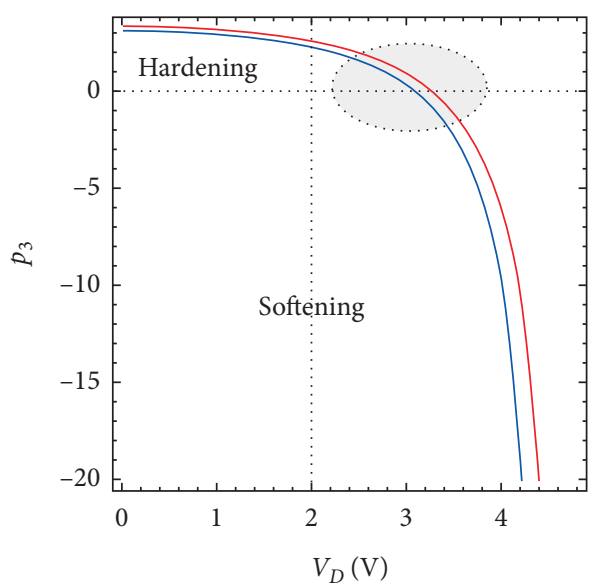

— Ideal boundary

- Nonideal boundary

(a)

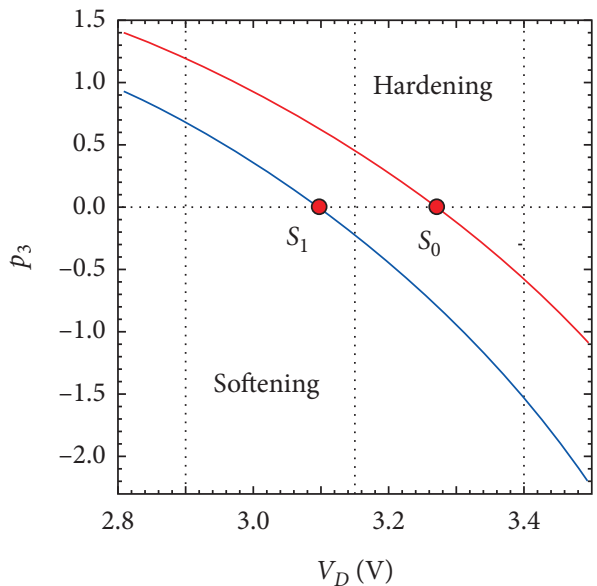

— Ideal boundary

- Nonideal boundary

(b)

FiguRE 8: Key parameters of local soft and hard characteristics. (a) Relationship between parameter $p_{3}$ and DC voltage under different boundary conditions. (b) Partial enlarged detail.

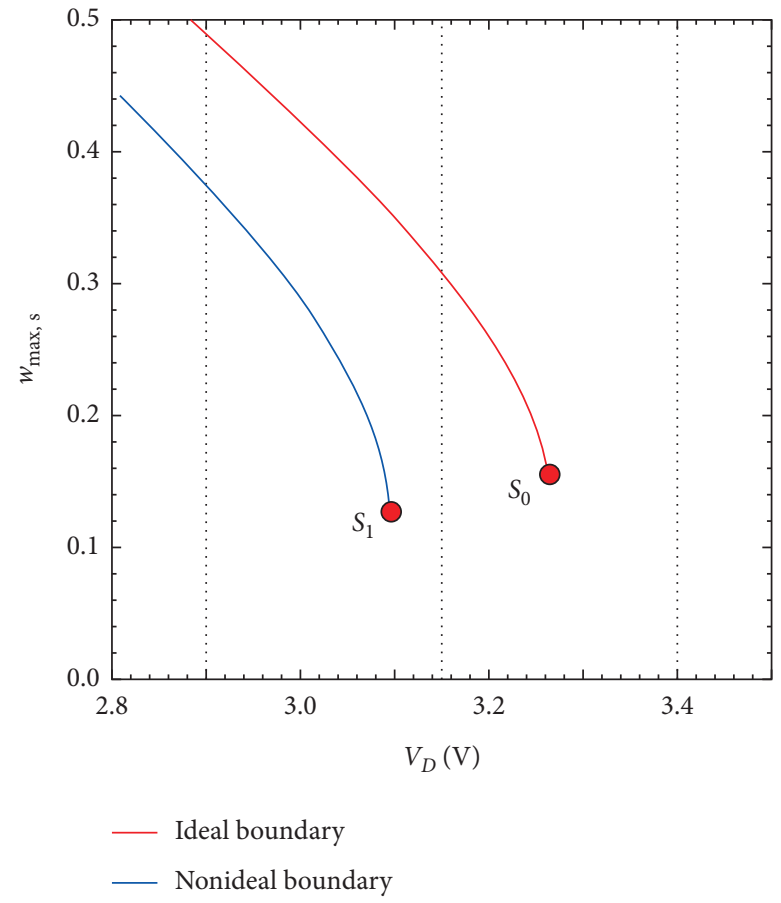

FIgURE 9: Maximum deflection at the time of the transition-DC voltage curve. 


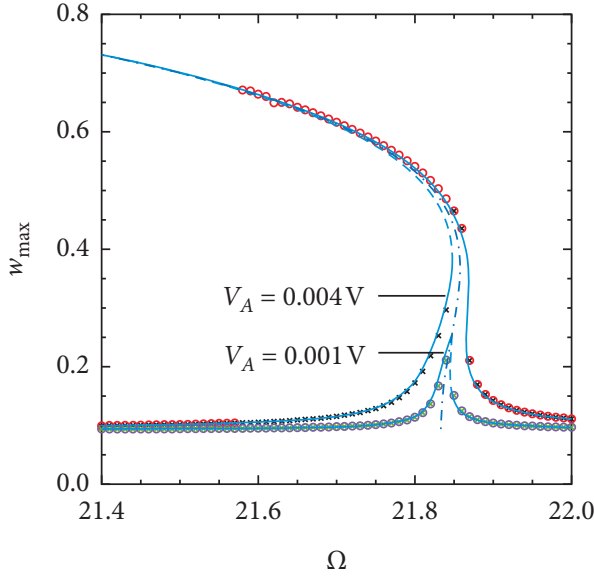

(a)

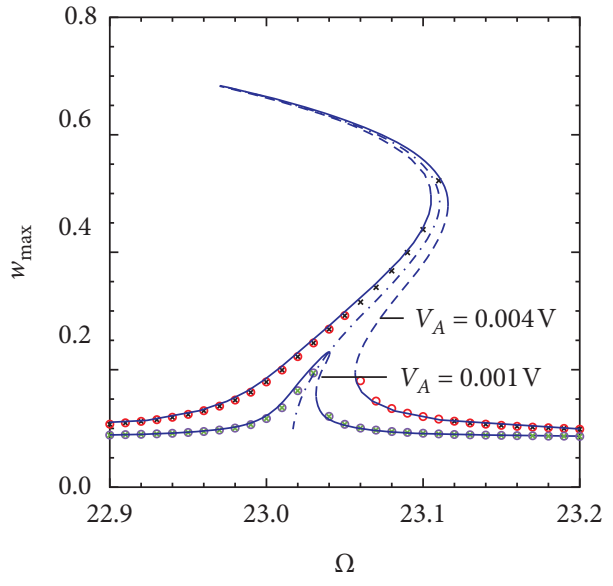

(b)

Figure 10: Amplitude-frequency curve: $V_{\mathrm{D}}=2.9 \mathrm{~V}$. (a) nonideal; (b) ideal.

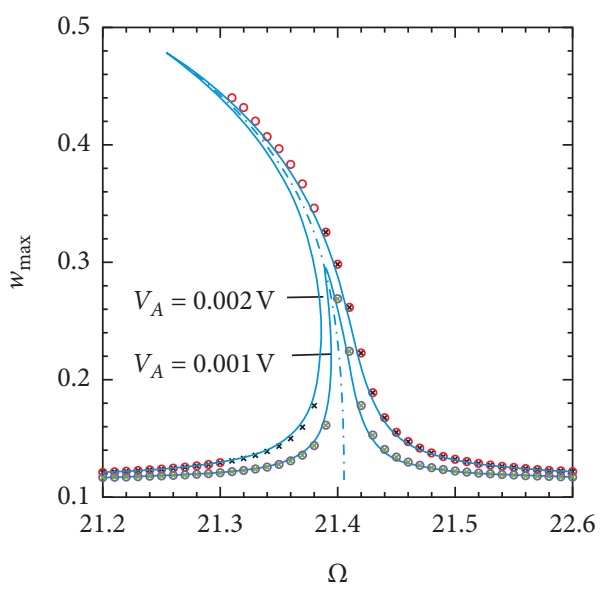

(a)

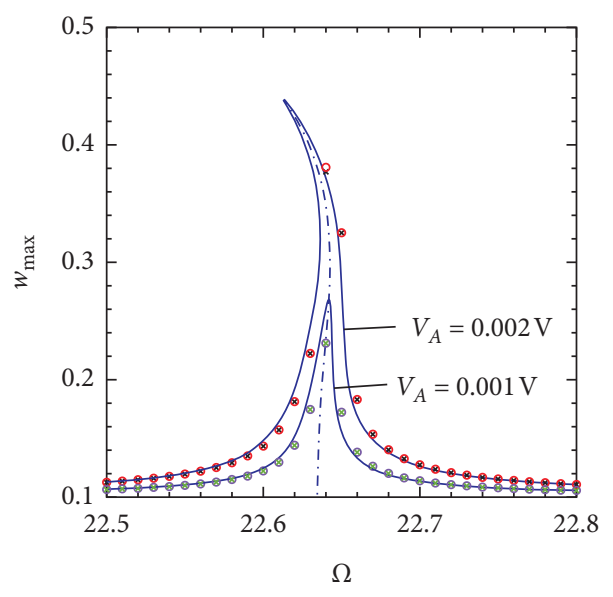

(b)

FIgURE 11: Amplitude-frequency curve: $V_{\mathrm{D}}=3.15 \mathrm{~V}$. (a) nonideal; (b) ideal.

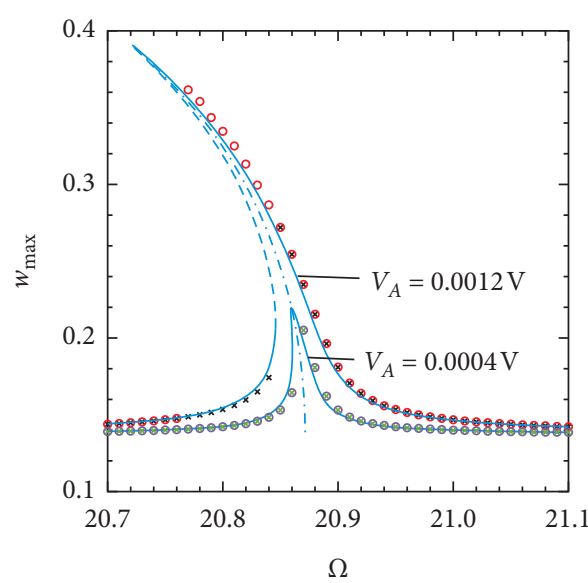

(a)

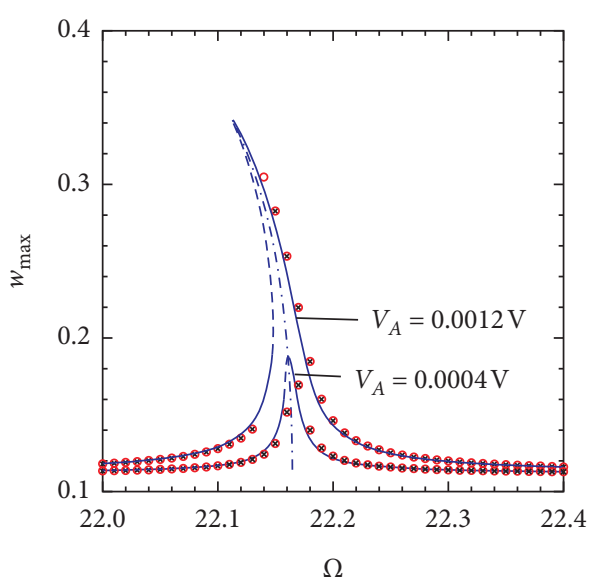

(b)

Figure 12: Amplitude-frequency curve: $V_{\mathrm{D}}=3.4 \mathrm{~V}$. (a) nonideal; (b) ideal. 
(i) Under the same DC voltage, as the spring stiffness goes down, the vibration frequency of the system becomes smaller and the amplitude becomes larger.

(ii) If the real threshold $a_{\mathrm{s}}$ does not exist, there will be no hardening-softening transition. If the real threshold $a_{\mathrm{s}}$ exists and $a_{s} \leq a_{\max }$ is satisfied, the system will have a hardening-softening transition phenomenon.

(iii) If there is hardening-softening transition, the nonideal boundary reduces the critical deflection of the transition. So, under the same DC and AC voltage, the transition occurs first.

(2) Case 3. In order to study the influence of translational spring on the system's softening and hardening characteristics, the spring stiffness coefficient values are taken as follows: $\kappa_{\mathrm{R}}=10^{50}, \kappa_{\mathrm{T}}=10^{3}, 10^{4}$. Similarly, select the three groups $V_{\mathrm{D}}$ to analyze the amplitude-frequency response. They are $1.5 \mathrm{~V}, 2.8 \mathrm{~V}$, and $3.4 \mathrm{~V}$, respectively.

When $\kappa_{\mathrm{T}}=10^{3}, V_{\mathrm{D}}=1.5 \mathrm{~V}$, the real threshold $a_{\mathrm{s}}$ exists, but does not meet with (19), and the system is in the hardening effect region. So, the system shows hardening characteristic, as shown in Figure 13(a). Likewise, the system with $\kappa_{\mathrm{T}}=10^{4}$ also shows hardening characteristics, as shown in Figure 13(b).

When $\kappa_{\mathrm{T}}=10^{3}, V_{\mathrm{D}}=2.8 \mathrm{~V}$, the real threshold $a_{\mathrm{s}}$ does not exist and the system is in softening effect region. So the system shows softening characteristic, as shown in Figure 14(a). However, the real threshold $a_{\mathrm{s}}$ of the system with $\kappa_{\mathrm{T}}=10^{4}$ exists and the system is in hardening effect region. So there is hardening-softening conversion if a suitable $V_{\mathrm{A}}$ to meet with the condition of (19) is selected, as shown in Figure 14(b).

When $\kappa_{\mathrm{T}}=10^{3}, V_{\mathrm{D}}=3.4 \mathrm{~V}$, the real threshold $a_{\mathrm{s}}$ does not exist and the system is in the softening effect region. So the system shows softening characteristic, as shown in Figure 15(a). Likewise, the system with $\kappa_{\mathrm{T}}=10^{4}$ also shows softening characteristics, as shown in Figure 15(b).

In a word, the laws of the dynamic performance of the system with the translational spring stiffness coefficient are the same as that of the comparison of Case 1 and Case 2 .

(3) Case 4. In order to study the influence of rotational spring on the system's softening and hardening characteristics, the spring stiffness coefficient values are taken as follows: $\kappa_{\mathrm{T}}=10^{50}, \kappa_{\mathrm{R}}=1,5$. Similarly, select the three groups $V_{\mathrm{D}}$ to analyze the amplitude-frequency response. They are $2 \mathrm{~V}$, $2.6 \mathrm{~V}$, and $2.75 \mathrm{~V}$, respectively.

When $\kappa_{\mathrm{R}}=1, V_{\mathrm{D}}=1.5 \mathrm{~V}$ the real threshold $a_{\mathrm{s}}$ exists and the system is in the hardening effect region. So there is hardening-softening conversion if a suitable $V_{\mathrm{A}}$ to meet with the condition of (19) is selected, as shown in Figure 16(a). Likewise, the system with $\kappa_{\mathrm{R}}=5$ shows hardening-softening conversion if a suitable $V_{\mathrm{A}}$ to meet

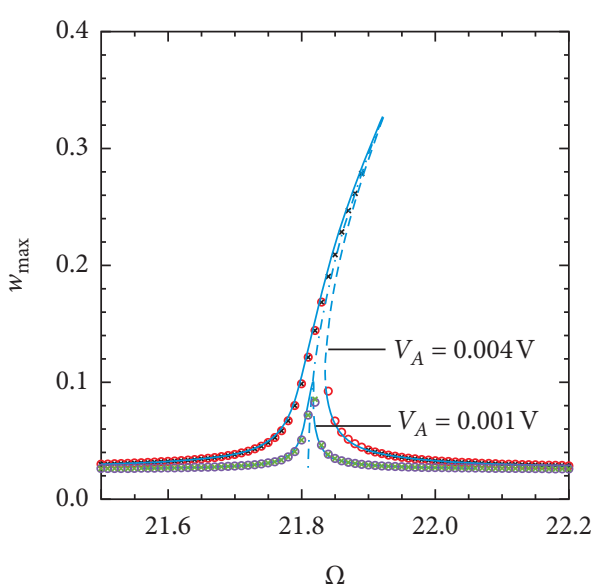

(a)

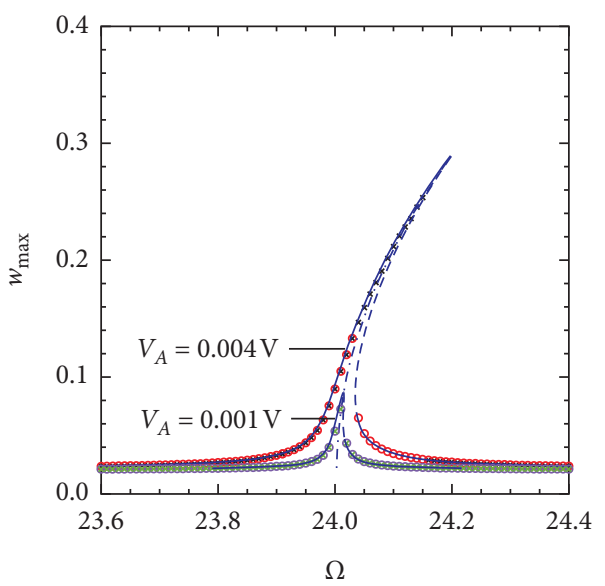

(b)

Figure 13: Amplitude-frequency curve: $V_{\mathrm{D}}=1.5 \mathrm{~V}$. (a) $\kappa_{\mathrm{T}}=10^{3}$; (b) $\kappa_{\mathrm{T}}=10^{4}$.

with the condition of (19) is selected, for example $V_{\mathrm{A}}=0.006 \mathrm{~V}$. However, because $V_{\mathrm{A}}$ selected in Figure 16(b) is too small, the system with $\kappa_{\mathrm{R}}=5$ only shows the hardening characteristic.

When $\kappa_{\mathrm{R}}=1, V_{\mathrm{D}}=2.6 \mathrm{~V}$, the real threshold $a_{\mathrm{s}}$ does not exist and the system is in the softening effect region. So the system shows softening characteristics, as shown in Figure 17(a). However, the real threshold $a_{\mathrm{s}}$ of the system with $\kappa_{\mathrm{R}}=5$ exists and the system is in the hardening effect region. So there is hardening-softening conversion if a suitable $V_{\mathrm{A}}$ to meet with the condition of (19) is selected, as shown in Figure 17(b).

When $\kappa_{\mathrm{R}}=1, V_{\mathrm{D}}=2.75 \mathrm{~V}$, the real threshold $a_{\mathrm{s}}$ does not exist and the system is in the softening effect region. So the system shows softening characteristic, as shown in Figure 18(a). Likewise, the system with $\kappa_{\mathrm{R}}=5$ also shows softening characteristics, as shown in Figure 18(b).

In a word, the laws of the dynamic performance of the system with the rotational spring stiffness coefficient are the same as that of the comparison of Case 1 and Case 2, too. 


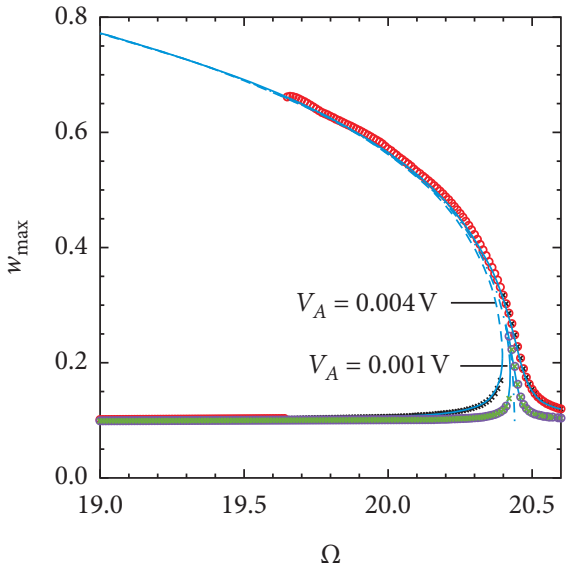

(a)

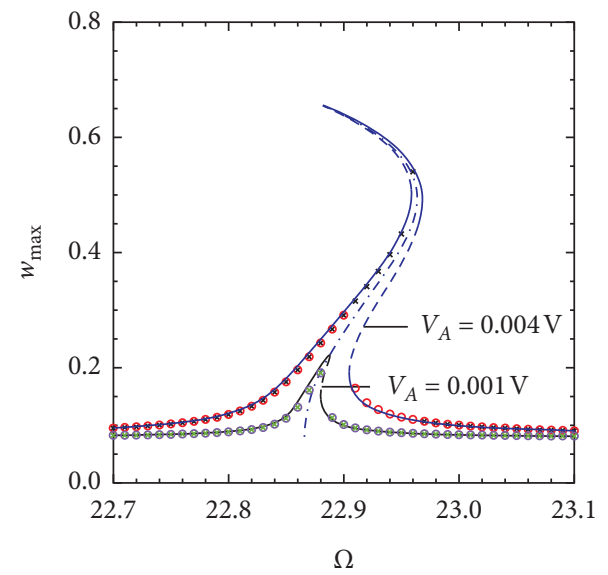

(b)

FIgURe 14: Amplitude-frequency curve: $V_{\mathrm{D}}=2.8 \mathrm{~V}$. (a) $\kappa_{\mathrm{T}}=10^{3}$; (b) $\kappa_{\mathrm{T}}=10^{4}$.

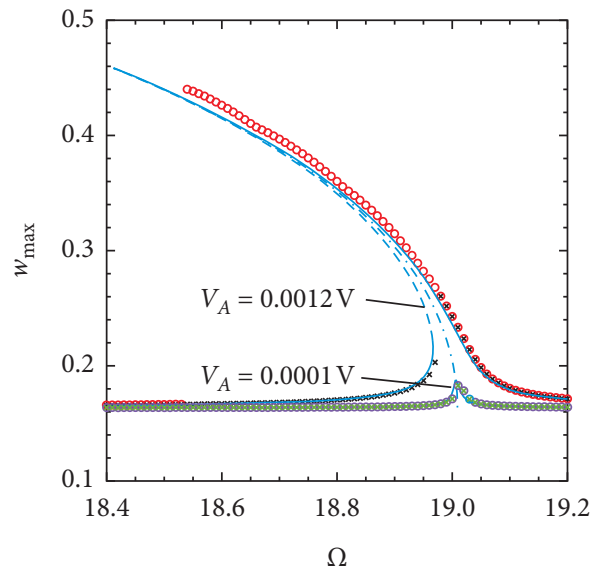

(a)

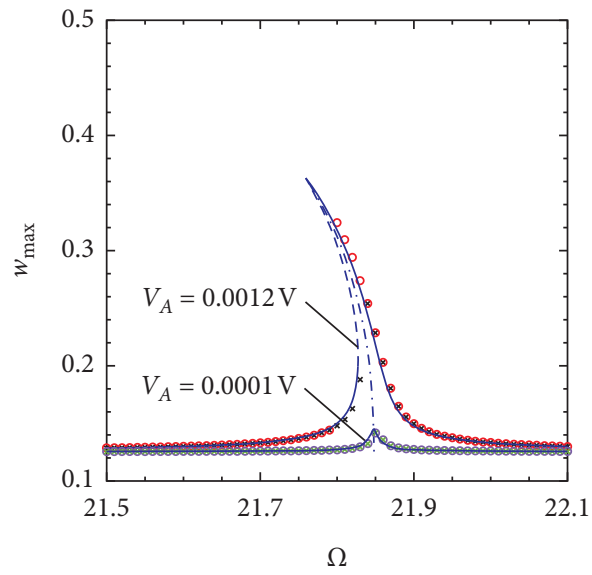

(b)

Figure 15: Amplitude-frequency curve: $V_{\mathrm{D}}=3.4 \mathrm{~V}$. (a) $\kappa_{\mathrm{T}}=10^{3}$; (b) $\kappa_{\mathrm{T}}=10^{4}$.

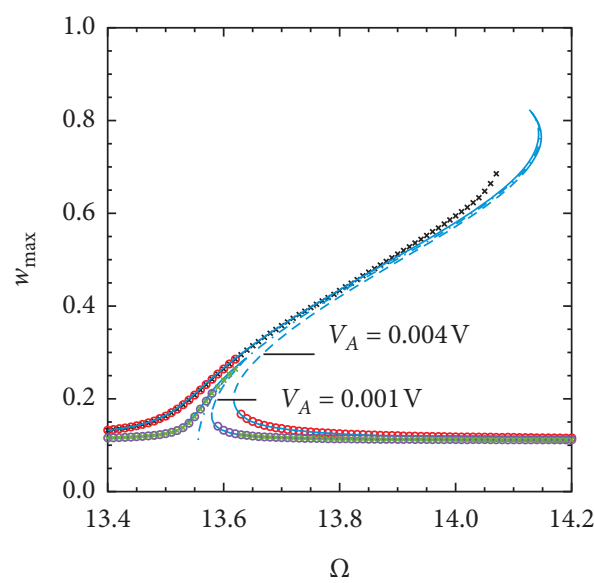

(a)

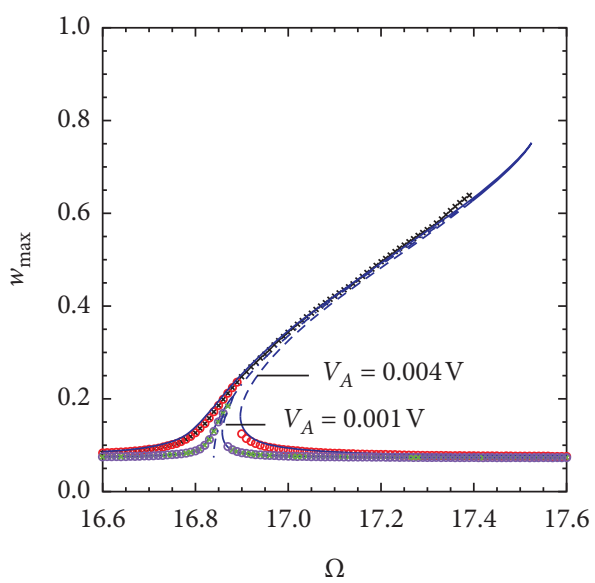

(b)

Figure 16: Amplitude-frequency curve: $V_{\mathrm{D}}=2 \mathrm{~V}$. (a) $\kappa_{\mathrm{R}}=1$; (b) $\kappa_{\mathrm{R}}=5$. 


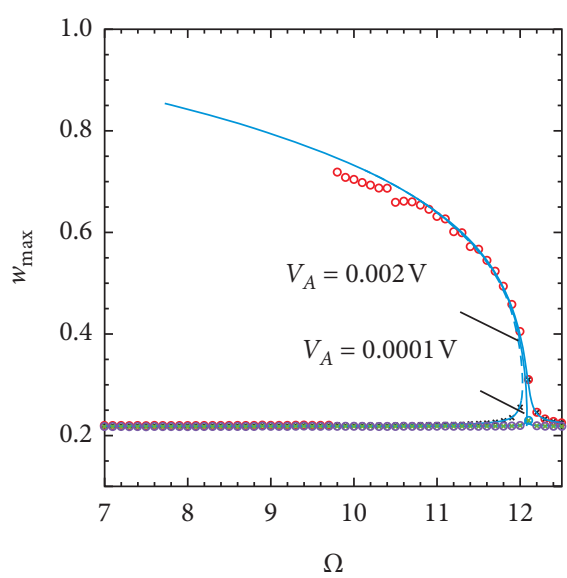

(a)

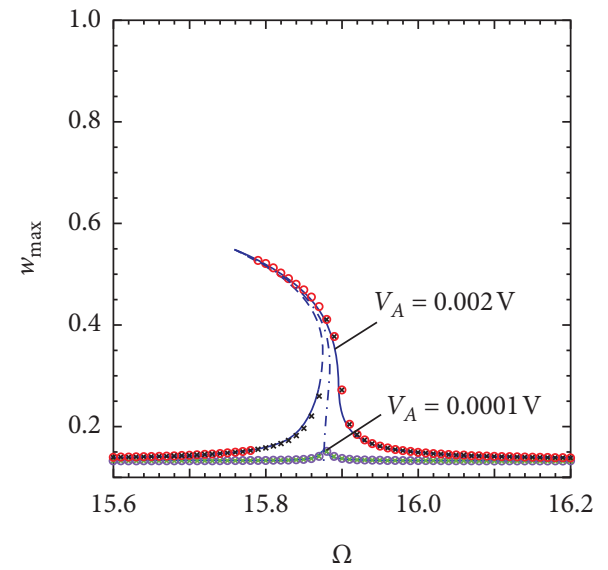

(b)

FIgURE 17: Amplitude-frequency curve: $V_{\mathrm{D}}=2.6 \mathrm{~V}$. (a) $\kappa_{\mathrm{R}}=1$; (b) $\kappa_{\mathrm{R}}=5$.

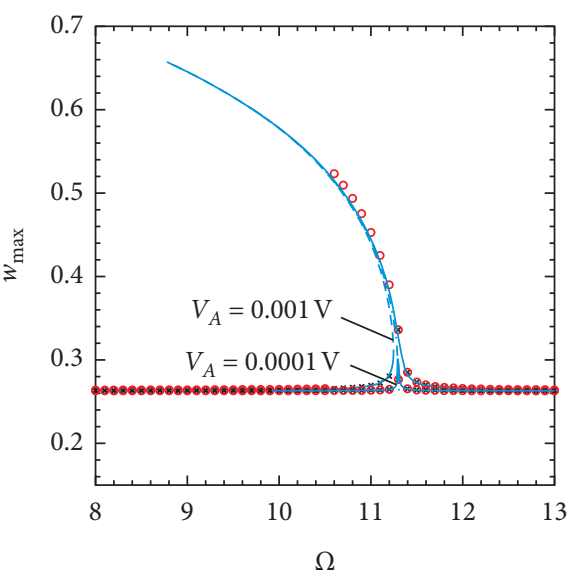

(a)

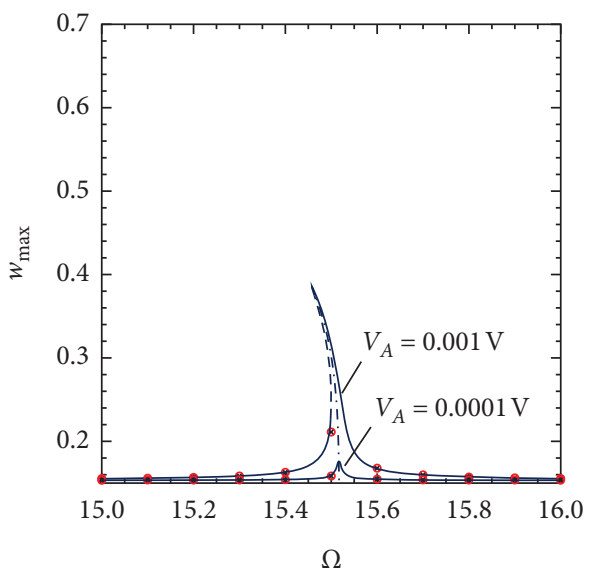

(b)

FIGURE 18: Amplitude-frequency curve: $V_{\mathrm{D}}=2.75 \mathrm{~V}$. (a) $\kappa_{\mathrm{R}}=1 ;$ (b) $\kappa_{\mathrm{R}}=5$.

\section{Conclusions}

In this paper, the Galerkin method and the method of multiple scales are used to theoretically investigate the mechanical behavior of electrostatic microbeam resonator with nonideal supports under forced excitation and study the feasibility of increasing vibration amplitude and reducing excitation voltage. The following main conclusions can be drawn.

(i) The laws of the system's static performances with the spring stiffness coefficients are obtained; with the growth of support stiffness before pull-in phenomenon occurs, the static deflection corresponding to the same voltage goes down, the static pull-in voltage increases, and eventually reaches a stable value.

(ii) The laws of the system's dynamic performances with the spring stiffness coefficients are obtained. Under the same DC voltage, as the support stiffness decreases, the vibration frequency of the system becomes smaller and the amplitude becomes larger.

(iii) The conditions for judging the hardening-softening transition are derived. If the real threshold $a_{\mathrm{s}}$ does not exist, there will be no hardening-softening transition. If the real threshold $a_{\mathrm{s}}$ exists and $a_{\mathrm{s}} \leq a_{\max }$ is satisfied, the system will have a hardening-softening transition phenomenon.

(iv) If there is hardening-softening transition, the nonideal boundary reduces the critical deflection of the transition. So, under the same DC and AC voltage, the transition occurs first.

The analysis provides a theoretical basis for implementing energy-saving resonators by adjusting the support stiffness values. The dynamic design of specific parameters can be further carried out to achieve frequency, amplitude regulation, and vibration form regulation.

So far, many studies have focused on the effect of Casimir gravity and size effect on the mechanical behavior of 
the system. The results show that both of them have a significant influence on the static and dynamic performance of the system. Then, the size effect on the system with nonideal boundary and Casimir force can be our next concern.

\section{Appendix}

\section{A. Mode Shapes of Microbeams with Nonideal Supports}

The mode shape of the linear undamped straight beam is expressed by the following equation:

$$
\varphi(x)=\cosh (e x)+R_{b} \sinh (e x)+R_{c} \cos (f x)+R_{d} \sin (f x),
$$

where

$$
\begin{aligned}
& e=\sqrt{\sqrt{\left(\frac{N}{2}\right)^{2}+\omega^{2}}+\frac{N}{2}} \\
& f=\sqrt{\sqrt{\left(\frac{N}{2}\right)^{2}+\omega^{2}}-\frac{N}{2}}, \\
& R_{b}=\frac{\left[\begin{array}{c}
-e^{2}\left(f^{4}+N f^{2}+\kappa_{\mathrm{R}} \kappa_{\mathrm{T}}\right) \cosh (e)+\left(e^{2} f^{4}+N e^{2} f^{2}-\kappa_{\mathrm{R}} \kappa_{\mathrm{T}} e^{2}-2 \kappa_{\mathrm{R}} \kappa_{\mathrm{T}} f^{2}\right) \cos (f) \\
-\kappa_{\mathrm{R}} e\left(f^{4}+N f^{2}+\kappa_{\mathrm{R}} \kappa_{\mathrm{T}}\right) \sinh (e)+f\left(-\kappa_{\mathrm{R}}^{2} \kappa_{\mathrm{T}}+\kappa_{\mathrm{R}} e^{2} f^{2}+N \kappa_{\mathrm{R}} e^{2}+\kappa_{\mathrm{T}} e^{2}+\kappa_{\mathrm{T}} f^{2}\right) \sin (f)
\end{array}\right]}{\left[\begin{array}{c}
\kappa_{\mathrm{R}} e\left(f^{4}+N f^{2}+\kappa_{\mathrm{R}} \kappa_{\mathrm{T}}\right) \cosh (e)-\kappa_{\mathrm{R}} e\left(-2 e^{2} f^{2}-f^{4}+N f^{2}+\kappa_{\mathrm{R}} \kappa_{\mathrm{T}}\right) \cos (f) \\
+e^{2}\left(f^{4}+N f^{2}+\kappa_{\mathrm{R}} \kappa_{\mathrm{T}}\right) \sinh (e)+e f\left(\kappa_{\mathrm{R}}^{2} e^{2}+\kappa_{\mathrm{R}}^{2} f^{2}+\kappa_{\mathrm{R}} \kappa_{\mathrm{T}}-e^{2} f^{2}+N f^{2}\right) \sin (f)
\end{array}\right]}, \\
& R_{c}=\frac{\left[\begin{array}{c}
\kappa_{\mathrm{R}}\left(e^{4}+2 e^{2} f^{2}+N e^{2}-\kappa_{\mathrm{R}} \kappa_{\mathrm{T}}\right) \cosh (e)+\kappa_{\mathrm{R}}\left(e^{4}-N e^{2}+\kappa_{\mathrm{R}} \kappa_{\mathrm{T}}\right) \cos (f) \\
+e\left(\kappa_{\mathrm{R}}^{2} e^{2}+\kappa_{\mathrm{R}}^{2} f^{2}-\kappa_{\mathrm{R}} \kappa_{\mathrm{T}}+e^{2} f^{2}+N e^{2}\right) \sinh (e)-f\left(e^{4}-N e^{2}+\kappa_{\mathrm{R}} \kappa_{\mathrm{T}}\right) \sin (f)
\end{array}\right]}{\left[\begin{array}{c}
\kappa_{\mathrm{R}}\left(f^{4}+N f^{2}+\kappa_{\mathrm{R}} \kappa_{\mathrm{T}}\right) \cosh (e)-\kappa_{\mathrm{R}}\left(-2 e^{2} f^{2}-f^{4}+N f^{2}+\kappa_{\mathrm{R}} \kappa_{\mathrm{T}}\right) \cos (f) \\
+e\left(f^{4}+N f^{2}+\kappa_{\mathrm{R}} \kappa_{\mathrm{T}}\right) \sinh (e)+f\left(\kappa_{\mathrm{R}}^{2} e^{2}+\kappa_{\mathrm{R}}^{2} f^{2}+\kappa_{\mathrm{R}} \kappa_{\mathrm{T}}-e^{2} f^{2}+N f^{2}\right) \sin (f)
\end{array}\right]}, \\
& R_{d}=\frac{\left[\begin{array}{c}
\left(-e^{4} f^{2}+N e^{2} f^{2}+2 \kappa_{\mathrm{R}} \kappa_{\mathrm{T}} e^{2}+\kappa_{\mathrm{R}} \kappa_{\mathrm{T}} f^{2}\right) \cosh (e)+f^{2}\left(e^{4}-N e^{2}+\kappa_{\mathrm{R}} \kappa_{\mathrm{T}}\right) \cos (f) \\
+e\left(\kappa_{\mathrm{R}}^{2} \kappa_{\mathrm{T}}-\kappa_{\mathrm{R}} e^{2} f^{2}+N \kappa_{\mathrm{R}} f^{2}+\kappa_{\mathrm{T}} e^{2}+\kappa_{\mathrm{T}} f^{2}\right) \sinh (e)+\kappa_{\mathrm{R}} f\left(e^{4}-N e^{2}+\kappa_{\mathrm{R}} \kappa_{\mathrm{T}}\right) \sin (f)
\end{array}\right]}{\left[\begin{array}{c}
\kappa_{\mathrm{R}} f\left(f^{4}+N f^{2}+\kappa_{\mathrm{R}} \kappa_{\mathrm{T}}\right) \cosh (e)-\kappa_{\mathrm{R}} f\left(-2 e^{2} f^{2}-f^{4}+N f^{2}+\kappa_{\mathrm{R}} \kappa_{\mathrm{T}}\right) \cos (f) \\
+e f\left(f^{4}+N f^{2}+\kappa_{\mathrm{R}} \kappa_{\mathrm{T}}\right) \sinh (e)+f^{2}\left(\kappa_{\mathrm{R}}^{2} e^{2}+\kappa_{\mathrm{R}}^{2} f^{2}+\kappa_{\mathrm{R}} \kappa_{\mathrm{T}}-e^{2} f^{2}+N f^{2}\right) \sin (f)
\end{array}\right]} \\
& \ddot{u}+\varepsilon^{4} \mu \dot{u}+k_{1} u+k_{2} u^{2}+k_{3} u^{3}+k_{4} u^{4}+k_{5} u^{5}=\varepsilon^{5} f_{1} \sin (\Omega t) .
\end{aligned}
$$

Eventually, each mode shape needs to be normalized by multiplying it by the constant $\xi_{i}$,

$$
\phi_{i}(x)=\xi_{i} \varphi_{i}(x),
$$

where $\xi_{i}$ makes the maximum value of the corresponding mode shape equal to 1 .

\section{B. Solving by the Method of Multiple Scales}

Considering the terms $V_{\mathrm{D}}=O(1), V_{\mathrm{A}}=O\left(\varepsilon^{5}\right)$, scale the damping and the dynamic forcing terms as follows:
Seek an approximate solution to this equation by letting

$$
\begin{aligned}
u(t ; \varepsilon)= & \varepsilon u_{1}\left(T_{0}, T_{1}, T_{2}, T_{3}, T_{4}\right)+\varepsilon^{2} u_{2}\left(T_{0}, T_{1}, T_{2}, T_{3}, T_{4}\right) \\
& +\varepsilon^{3} u_{3}\left(T_{0}, T_{1}, T_{2}, T_{3}, T_{4}\right)+\varepsilon^{4} u_{4}\left(T_{0}, T_{1}, T_{2}, T_{3}, T_{4}\right) \\
& +\varepsilon^{5} u_{5}\left(T_{0}, T_{1}, T_{2}, T_{3}, T_{4}\right)+\cdots
\end{aligned}
$$

where $T_{n}=\varepsilon^{n} t,(n=0,1,2,3,4)$. 
Introducing the following operator:

$$
\begin{aligned}
\frac{\mathrm{d}}{\mathrm{d} t}= & \varepsilon^{0} D_{0}+\varepsilon^{1} D_{1}+\varepsilon^{2} D_{2}+\varepsilon^{3} D_{3}+\varepsilon^{4} D_{4}+\varepsilon^{5} D_{5}+\cdots, \\
\frac{\mathrm{d}^{2}}{\mathrm{~d} t^{2}}= & \varepsilon^{0} D_{0}^{2}+\varepsilon^{1} 2 D_{0} D_{1}+\varepsilon^{2}\left(D_{1}^{2}+2 D_{0} D_{2}\right) \\
& +\varepsilon^{3}\left(2 D_{1} D_{2}+2 D_{0} D_{3}\right) \\
& +\varepsilon^{4}\left(D_{2}^{2}+2 D_{1} D_{3}+2 D_{0} D_{4}\right) \\
& +\varepsilon^{5}\left(2 D_{2} D_{3}+2 D_{1} D_{4}+2 D_{0} D_{5}\right)+\cdots
\end{aligned}
$$

where $D_{i}=\partial / \partial T_{i}, i=0,1, \ldots$

Introducing a tuning parameter $\sigma$ and letting $\Omega=\omega_{n}+\varepsilon^{4} \sigma$, obtain the following governing equation:

$O\left(\varepsilon^{1}\right): D_{0}^{2} u_{1}+\omega_{n}^{2} u_{1}=0$,

$O\left(\varepsilon^{2}\right): D_{0}^{2} u_{2}+\omega_{n}^{2} u_{2}=-2 D_{0} D_{1} u_{1}-k_{2} u_{1}^{2}$,

$O\left(\varepsilon^{3}\right): D_{0}^{2} u_{3}+\omega_{n}^{2} u_{3}=-D_{1}^{2} u_{1}-2 D_{0} D_{2} u_{1}-k_{3} u_{1}^{3}-2 D_{0} D_{1} u_{2}-2 k_{2} u_{1} u_{2}$,

$O\left(\varepsilon^{4}\right): D_{0}^{2} u_{4}+\omega_{n}^{2} u_{4}=-2 D_{1} D_{2} u_{1}-2 D_{0} D_{3} u_{1}-k_{4} u_{1}^{4}-D_{1}^{2} u_{2}-2 D_{0} D_{2} u_{2}-3 k_{3} u_{1}^{2} u_{2}-k_{2} u_{2}^{2}-2 D_{0} D_{1} u_{3}-2 k_{2} u_{1} u_{3}$,

$O\left(\varepsilon^{5}\right): D_{0}^{2} u_{5}+\omega_{n}^{2} u_{5}=-D_{2}^{2} u_{1}-2 D_{1} D_{3} u_{1}-2 D_{0} D_{4} u_{1}-k_{5} u_{1}^{5}-2 D_{1} D_{2} u_{2}-2 D_{0} D_{3} u_{2}-4 k_{4} u_{1}^{3} u_{2}-3 k_{3} u_{1} u_{2}^{2}-D_{1}^{2} u_{3}$

$$
-2 D_{0} D_{2} u_{3}-3 k_{3} u_{1}^{2} u_{3}-2 k_{2} u_{2} u_{3}-2 D_{0} D_{1} u_{4}-2 k_{2} u_{1} u_{4}-\mu D_{0} u_{1}+f_{1} \sin \left(\omega_{n} T_{0}+\sigma T_{4}\right) \text {. }
$$

Eventually, the average equation can be given by the following equation:

$$
\left\{\begin{array}{l}
\frac{\mathrm{d} a}{\mathrm{~d} t}=-\frac{\mu a}{2}-\frac{f_{1} \cos \gamma}{2 \omega_{n}} \\
a \frac{\mathrm{d} \gamma}{\mathrm{d} t}=p_{1} a-p_{3} a^{3}-p_{5} a^{5}-\frac{f_{1} \sin \gamma}{2 \omega_{n}}
\end{array}\right.
$$

where $\gamma=\sigma T_{4}-\beta$ a represents amplitude, and $\beta$ represents phase.

The approximate dynamic solution can be given by the following:

$$
u=\sum_{i=0}^{5} \chi_{i} \cos \left[i\left(\omega_{n} t+\beta\right)\right]
$$

where

$$
\begin{aligned}
& \chi_{0}=\frac{-36 a^{2} k_{2} \omega_{n}^{4}+a^{4}\left(-19 k_{2}^{3}+45 k_{2} k_{3} \omega_{n}^{2}-27 k_{4} \omega_{n}^{4}\right)}{72 \omega_{n}^{6}}, \\
& \chi_{1}=a \\
& \chi_{2}=\frac{144 a^{2} k_{2} \omega_{n}^{4}+a^{4}\left(118 k_{2}^{3}-279 k_{2} k_{3} \omega_{n}^{2}+144 k_{4} \omega_{n}^{4}\right)}{864 \omega_{n}^{6}}, \\
& \chi_{3}=\frac{a^{3}\left[480\left(2 k_{2}^{2} \omega_{n}^{4}+3 k_{3} \omega_{n}^{6}\right)+a^{2}\left(1580 k_{2}^{4}-2580 k_{2}^{2} k_{3} \omega_{n}^{2}-945 k_{3}^{2} \omega_{n}^{4}-432 k_{2} k_{4} \omega_{n}^{4}+1800 k_{5} \omega_{n}^{6}\right)\right]}{46080 \omega_{n}^{8}}, \\
& \chi_{4}=\frac{a^{4}\left(10 k_{2}^{3}+45 k_{2} k_{3} \omega_{n}^{2}+36 k_{4} \omega_{n}^{4}\right)}{4320 \omega_{n}^{6}}, \\
& \chi_{5}=\frac{a^{5}\left[100 k_{2}^{4}+900 k_{2}^{2} k_{3} \omega_{n}^{2}+1584 k_{2} k_{4} \omega_{n}^{4}+135\left(3 k_{3}^{2} \omega_{n}^{4}+8 k_{5} \omega_{n}^{6}\right)\right]}{414720 \omega_{n}^{8}} .
\end{aligned}
$$




\section{Data Availability}

The data used to support the findings of this study are included within the article, see Table 1 for details.

\section{Conflicts of Interest}

The authors declare that there are no conflicts of interest regarding the publication of this paper.

\section{Acknowledgments}

The authors are grateful for the support from the National Natural Science Foundation of China (Grant nos. 11872044, 11702192 , and 11772218).

\section{References}

[1] A. Bouchaala, A. H. Nayfeh, N. Jaber, and M. I. Younis, "Mass and position determination in mems mass sensors: a theoretical and an experimental investigation," Journal of Micromechanics and Microengineering, vol. 26, no. 10, p. 11, 2016.

[2] M. Jrad, M. I. Younis, and F. Najar, "Modeling and design of an electrically actuated resonant microswitch," Journal of Vibration and Control, vol. 22, no. 2, pp. 559-569, 2016.

[3] M. I. Younis and A. H. Nayfeh, "A study of the nonlinear response of a resonant microbeam to an electric actuation," Nonlinear Dynamics, vol. 31, no. 1, pp. 91-117, 2003.

[4] A. H. Nayfeh, M. I. Younis, and E. M. Abdel-Rahman, "Dynamic pull-in phenomenon in mems resonators," Nonlinear Dynamics, vol. 48, no. 1-2, pp. 153-163, 2007.

[5] J. Han, G. Jin, Q. Zhang et al., "Dynamic evolution of a primary resonance mems resonator under prebuckling pattern," Nonlinear Dynamics, vol. 93, no. 4, pp. 2357-2378, 2018.

[6] L. Li, Q. Zhang, W. Wang, and J. Han, "Dynamic analysis and design of electrically actuated viscoelastic microbeams considering the scale effect," International Journal of Non-linear Mechanics, vol. 90, pp. 21-31, 2017.

[7] J. Han, Q. Zhang, and W. Wang, "Static bifurcation and primary resonance analysis of a mems resonator actuated by two symmetrical electrodes," Nonlinear Dynamics, vol. 80, no. 3, pp. 1585-1599, 2015.

[8] D. Younesian, M. Sadri, and E. Esmailzadeh, "Primary and secondary resonance analyses of clamped-clamped microbeams," Nonlinear Dynamics, vol. 76, no. 4, pp. 1867-1884, 2014.

[9] N. Kacem, S. Baguet, R. Dufour, and S. Hentz, "Stability control of nonlinear micromechanical resonators under simultaneous primary and superharmonic resonances," Applied Physics Letters, vol. 98, no. 19, p. 193507, 2011.

[10] B. Akgöz and Ö. Civalek, "Effects of thermal and shear deformation on vibration response of functionally graded thick composite microbeams," Composites Part B: Engineering, vol. 129, pp. 77-87, 2017.

[11] R. C. Batra, M. Porfiri, and D. Spinello, "Review of modeling electrostatically actuated microelectromechanical systems," Smart Materials and Structures, vol. 16, no. 6, pp. R23-R31, 2007.

[12] Ç. Demir and Ö. Civalek, "On the analysis of microbeams," International Journal of Engineering Science, vol. 121, p. 20, 2017.
[13] M. Lishchynska, N. Cordero, and O. S. Nmrc, "State of the art in prediction of mechanical behaviour of microsystems," IEEE, vol. 121, 2004.

[14] H. M. Numanoğlu, B. Akgöz, and Ö. Civalek, "On dynamic analysis of nanorods," International Journal of Engineering Science, vol. 130, p. 18, 2018.

[15] M. I. Younis, "Analytical expressions for the electrostatically actuated curled beam problem," Microsystem Technologies, vol. 21, no. 8, pp. 1709-1717, 2014.

[16] B. Akgöz and Ö. Civalek, "Longitudinal vibration analysis for microbars based on strain gradient elasticity theory," Journal of Vibration and Control, vol. 20, no. 4, pp. 606-616, 2012.

[17] M. Fathalilou, M. Sadeghi, and G. Rezazadeh, "Micro-inertia effects on the dynamic characteristics of micro-beams considering the couple stress theory," Mechanics Research Communications, vol. 60, no. 7, 2014.

[18] A. Koochi, A. S. Kazemi, Y. Tadi Beni, A. Yekrangi, and M. Abadyan, "Theoretical study of the effect of casimir attraction on the pull-in behavior of beam-type nems using modified adomian method," Physica E: Low-Dimensional Systems and Nanostructures, vol. 43, no. 2, pp. 625-632, 2010.

[19] Y. Tadi Beni, M. Abadyan, and A. Koochi, "Effect of the casimir attraction on the torsion/bending coupled instability of electrostatic nano-actuators," Physica Scripta, vol. 84, no. 6, 2011.

[20] M. Ö. Yayli, M. Aras, and S. Aksoy, "Free vibration behavior of a gradient elastic beam with varying cross section," Shock and Vibration, vol. 2014, pp. 1-11, 2014.

[21] Z. Wang and R. Li, "Transverse vibration of rotating tapered cantilever beam with hollow circular cross-section," Shock and Vibration, vol. 2018, p. 14, 2018.

[22] G. Rinaldi, M. Packirisamy, and I. Stiharu, "Quantitative boundary support characterization for cantilever mems," Sensors, vol. 7, no. 10, pp. 2062-2079, 2007.

[23] G. Sari and M. Pakdemirli, "Vibrations of a slightly curved microbeam resting on an elastic foundation with nonideal boundary conditions," Mathematical Problems in Engineering, vol. 7, 2013.

[24] S. A. Alkharabsheh and M. I. Younis, "Dynamics of mems arches of flexible supports," Journal of Microelectromechanical Systems, vol. 22, no. 1, pp. 216-224, 2013.

[25] H. M. Ouakad and M. I. Younis, "The dynamic behavior of mems arch resonators actuated electrically," International Journal of Non-linear Mechanics, vol. 45, no. 7, pp. 704-713, 2010.

[26] S. A. Alkharabsheh and M. I. Younis, "Statics and dynamics of mems arches under axial forces," Journal of Vibration and Acoustics-Transactions of the Asme, vol. 135, no. 2, 2013.

[27] D. V. Bambill, G. I. Guerrero, and D. H. Felix, "Natural vibrations of micro beams with nonrigid supports," Journal of Vibration and Control, vol. 23, no. 19, pp. 3233-3246, 2016.

[28] P. Muthukumaran, R. B. Bhat, and I. Stiharu, "Boundary conditioning technique for structural tuning," Journal of Sound and Vibration, vol. 220, p. 13, 1999.

[29] M. Pallay and S. Towfighian, "Parametrically excited electrostatic mems cantilever beam with flexible support," Journal of Vibration and Acoustics-Transactions of the Asme, vol. 139, no. 2, 2017.

[30] G. Rinaldi, M. Packirisamy, and I. Stiharu, "Boundary characterization of mems structures through electro-mechanical testing," Sensors and Actuators A: Physical, vol. 143, no. 2, pp. 415-422, 2008. 
[31] M. P. Rezaei and M. Zamanian, "A two-dimensional vibration analysis of piezoelectrically actuated microbeam with nonideal boundary conditions," Physica E, vol. 85, no. 9, 2017.

[32] Z.-Y. Zhong, W.-M. Zhang, and G. Meng, "Dynamic characteristics of micro-beams considering the effect of flexible supports," Sensors, vol. 13, no. 12, pp. 15880-15897, 2013.

[33] Z. Y. Zhong, J. P. Zhou, H. L. Zhang, and T. Zhang, "Effect of the equivalent stiffness of flexible supports on the mems cantilever-based sensors," Comput. Struct.vol. 169, p. 11, 2016.

[34] H. O. Ekici and H. Boyaci, "Effects of nonideal boundary conditions on vibrations of microbeams," Journal of Vibration and Control, vol. 13, no. 9-10, pp. 1369-1378, 2007.

[35] J. Zeng, A. Garg, A. Kovacs, A. K. Bajaj, and D. Peroulis, “An equation-based nonlinear model for non-flat mems fixed-fixed beams with non-vertical anchoring supports," Journal of Micromechanics and Microengineering, vol. 25, no. 5, 2015.

[36] S. Bashmal, Y. Khulief, and W. A. Oke, "Vibration analysis of an elastically restrained microcantilever beam under electrostatic loading using wavelet-based finite element method," Journal of Micromechanics and Microengineering, vol. 10, no. 3, pp. 147-152, 2015.

[37] W. Chuang, Y. Hu, C. Lee, W. Shih, and P. Chang, "Electromechanical behavior of the curled cantilever beam," Journal of Micromechanics and Microengineering.vol. 8, no. 3, 2009.

[38] M. Ö. Yayli, "A compact analytical method for vibration of micro-sized beams with different boundary conditions," Mechanics of Advanced Materials and Structures, vol. 24, no. 6, pp. 496-508, 2016.

[39] M. Lishchynska, N. Cordero, O. Slattery, and C. O'mahony, "Modelling electrostatic behaviour of microcantilevers incorporating residual stress gradient and nonideal anchors," Journal of Micromechanics and Microengineering, vol. 15, no. 7, p. 5, 2005.

[40] Y. Tadi Beni, A. Koochi, and M. Abadyan, "Theoretical study of the effect of casimir force, elastic boundary conditions and size dependency on the pull-in instability of beam-type nems," Physica E: Low-Dimensional Systems and Nanostructures, vol. 43, no. 4, pp. 979-988, 2011.

[41] M. Shojaeian, Y. T. Beni, and H. Ataei, "Size-dependent snapthrough and pull-in instabilities of initially curved prestressed electrostatic nano-bridges," Journal of Physics D-Applied Physics, vol. 49, no. 29, p. 15, 2016.

[42] M. I. Younis, Mems Linear and Nonlinear Statics and Dynamics, Springer Science + Business Media, New York, NY, USA, 2011.

[43] A. H. Nayfeh and D. T. Mook, Nonlinear Oscillations, John Wiley \& Sons, New York, NY, USA, 1979.

[44] G. Rinaldi, M. Packirisamy, and I. Stiharu, "Dynamic testing of micromechanical structures under thermo-electro-mechanical influences," Measurement, vol. 40, no. 6, pp. 563574, 2007.

[45] M. A. Attia and S. A. Mohamed, "Nonlinear modeling and analysis of electrically actuated viscoelastic microbeams based on the modified couple stress theory," Applied Mathematical Modelling, vol. 41, p. 28, 2017.

[46] V. M. Bhojawala and D. P. Vakharia, "Effect of van der waals force on pull-in voltage, frequency tuning and frequency stability of nems devices," Microsystem Technologies, vol. 23, no. 5, pp. 1255-1267, 2017.

[47] D. Wagg and S. Neild, "Nonlinear vibration with control for flexible and adaptive structures," 2015. 